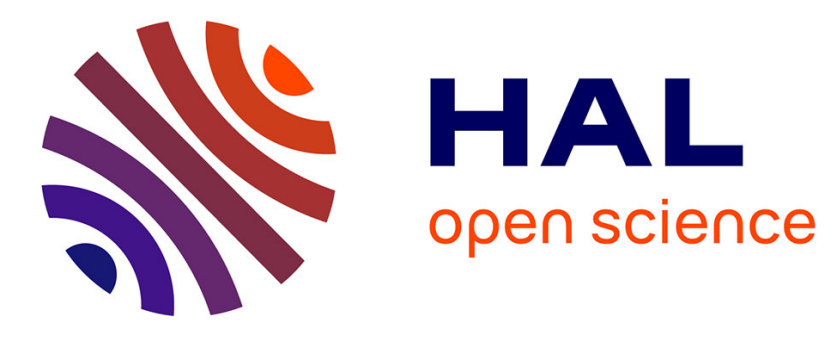

\title{
An interface model for homogenization of acoustic materials
}

\author{
Jean-Jacques Marigo, Agnes Maurel
}

\section{To cite this version:}

Jean-Jacques Marigo, Agnes Maurel. An interface model for homogenization of acoustic materials. Richard Craster, Sébastien Guenneau; Sébastien Guenneau. World Scientific Handbook of Metamaterials and Plasmonics, 2, World Scientific, Chapter 14, pp. 599-645, 2018, Elastic, Acoustic, and Seismic Metamaterials, 978-981-3227-63-7. 10.1142/9789813228702_0014 . hal-01699645

\section{HAL Id: hal-01699645 \\ https://hal-polytechnique.archives-ouvertes.fr/hal-01699645}

Submitted on 11 Jan 2022

HAL is a multi-disciplinary open access archive for the deposit and dissemination of scientific research documents, whether they are published or not. The documents may come from teaching and research institutions in France or abroad, or from public or private research centers.
L'archive ouverte pluridisciplinaire HAL, est destinée au dépôt et à la diffusion de documents scientifiques de niveau recherche, publiés ou non, émanant des établissements d'enseignement et de recherche français ou étrangers, des laboratoires publics ou privés.

\section{(ㄷ)(1) $\$$}

Distributed under a Creative Commons Attribution - NonCommerciall 4.0 International 


\section{An Interface Model for Homogenization of Acoustic Metafilms}

\section{JEAN-JACQUES MARIGO}

École Polytechnique, France

\section{AGNÈS MAUREL*}

Institut Langevin, France

\subsection{Introduction}

Acoustic metamaterials consisting in massive materials perforated by periodic subwavelength holes [Pendry et al. (2004); Garcia-Vidal et al. (2005); Zhu et al. (2011)] or more sparse structures involving periodic arrangement of wires [Lemoult et al. (2013)] have been shown to be able to control the wave propagation with high flexibility. In comparison, phononic crystals have a priori higher dimensions because of their wavelength-scale period. However, if the metamaterial has subwavelength period, many of the observed phenomena are attributable to Fabry-Perot type resonances, resonances in the hole or resonances of the wires. Therefore, these structures have a

*Corresponding author: agnes.maurel@espci.fr 
limitation in their thickness, which has to be at wavelength scale to produce such resonances (and thickness refers to the size in the direction perpendicular to the plane containing the periodic cells). In order to reduce the size of the devices, structures with subwavelength thickness have been developed. They are known as metasurfaces and metafilms. Despite the vanishing thickness in comparison to the incident wavelength, the capability of these ultrathin devices to control the wave propagation has been evidenced. This is because they are based on a resonance which is not related to their thickness. It can be a thin elastic membrane within the unit cell [Ma et al. (2014); Zhao et al. (2014)], or resonances of labyrinthine or curled elements squeezed in the unit cell [Peng et al. (2014); Li et al. (2014); Xie et al. (2014)].

Because of their subwavelength unit cell, homogenization techniques are natural tools to describe the effective properties of metamaterials. Standard homogenization methods [Zhu et al. (2013); Maurel et al. (2013)] or more empirical methods, as the retrieval techniques [Arslanagic et al. (2013); Castanié et al. (2014)] (often referred as Nicolson-Ross-Weir technique) and approximated modal approaches [Pendry et al. (2004); Garcia-Vidal et al. (2005); Kelders et al. (1998)] have been largely used for massive structures leading to effective bulk modulus and effective mass density, being the acoustic counterparts of the effective permittivity and permeability in electromagnetism. An enlightening review can be found in Ref. [Simovski (2011)]. When metafilms or metasurfaces are concerned, first attempts have been proposed which use the same retrieval parameters as for bulk materials and affecting a thickness value more or less relevant (see the discussions in $\S 3$ of [Simovski (2011)] and in $\S 2$ of [Holloway et al. (2009)]). It is now admitted that these approaches are not pertinent and rather, jump conditions or transmission conditions of the fields across a zero thickness surface are thought. In the context of electromagnetism, effective surface parameters are expressed in term of effective surface admittances [Zhao et al. (2011)] or surface susceptibilities [Holloway 
et al. (2009); Dimitriadis et al. (2012); Kuester et al. (2003); Holloway et al. (2005)]. In addition to be closely related to the notion of electric polarization density and surface magnetization, which are not easily transposable to the case of acoustic or elastic waves, these techniques require an inversion procedure to get the surface susceptibilities, from the measurement of the scattering coefficients. Thus, although the problem of the artificial thickness is avoided, the question of wether or not the obtained parameters depend on the scattering problem that has been considered remains.

In this paper, we present a homogenization method for vanishing thickness metamaterials which explicitly accounts for the microstructure of the unit cell. The problem ends with jump conditions for the acoustic pressure and for the normal acoustic velocity involving parameters being wave independent, by construction. This is because, as in the standard homogenization, the parameters are determined by solving (analytically or numerically) elementary cell problems in the static case (that is for zero frequency). The model relies on a separation of scales, a micro scale associated to the small scatterer size and a macro scale associated to the acoustic wavelength, and $\varepsilon$ is the small parameter that measures the ratio of the two scales. Each scale is associated to a system of coordinates which is relevant or not to describe the variation of the acoustic fields wether we are close to the film or far from it. Thus, a separation of the space is used, into an outer region, typically the far field, where only the macro scale makes sense and an inner region, the near field, where both the micro and macro scales are needed. Expansions of the fields in power of $\varepsilon$ are performed in both regions and finally, matching conditions are used between the two regions. This approach has been developed in the context of static elasticity, see [Marigo and Pideri (2011); David et al. (2012)] for a complete description. The case of wave propagation has been less regarded. We mention the works of Capdeville and Marigo for seismic waves in the time domain [Capdeville and Marigo (2007); Capdeville et al. (2010a); Guillot et al. (2010); 
Capdeville et al. (2010b); Capdeville and Marigo (2013)], and the Refs. [Bonnet-Bendhia et al. (2004); Delourme (2010); Delourme et al. (2012)] in the frequency domain. Note also works using alternative forms of the matched asymptotic expansions [Sanchez-Hubert and Sanchez-Palencia (1982); Martin and Dalrymple (1988); Kakuno et al. (1992)].

The paper is organized as follows. In Section 14.2, we apply the interface model for the problem of acoustic wave propagation through a thin film made of a periodic row of sound hard inclusions. The jump conditions are derived, Eqs. (14.27), with the interface parameters $(\mathbf{B}, \mathbf{C})$ being associated to the elementary problems, Eqs. (14.17). Section 14.3 presents results in the case of rectangular inclusions in two dimensions, with thickness $e$. This choice is not casual. First, it allows for approximate, but explicit, solutions of the elementary problems. Thus, the interface parameters $(\mathbf{B}, \mathbf{C})$ are given, and associated transmission and reflection coefficients of a plane wave at oblique incidence, Eqs. (14.33), are obtained. Next, a comparison with the classical homogenization of layered media is possible since again, explicit solutions are available in this case [Garcia-Vidal et al. (2005); Oleinik et al. (2009)], Eq. (14.34). The validity of our interface model is inspected by comparison with full wave simulations, and discussed in the light of the comparison with the classical homogenization. The results show that the interface homogenization is accurate for $k h, k e<1$, while classical homogenization can be used for $k h<1$ and $e>h$ and it largely underestimates the scattering properties of thin structured layers. The small parameter $\varepsilon$ can be forced to values of order unity; typically for $\varepsilon$ and $k e$ of order unity, the discrepancy between the exact solution and the solution of the homogenized problem do not exceed few per percent. We end with an illustrative example of an ultra thin structured film obstructing a waveguide with rigid walls. Again, an explicit solution of the homogenized problem is possible and a good agreement with direct numerics is obtained. Technical calculations are collected in the Appendices. 


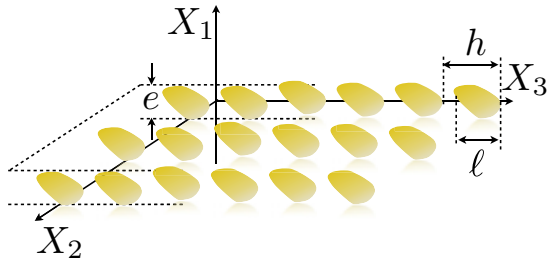

(a)

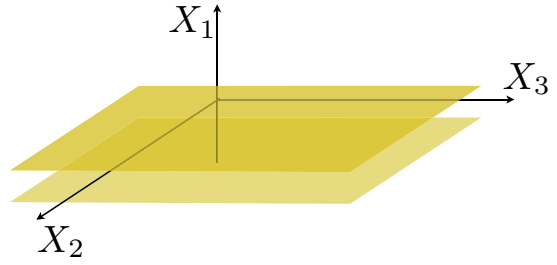

(b)

Fig. 14.1. Scattering problem for (a) the microstructured film (metafilm) and

(b) the equivalent interface. $\mathbf{X}=\left(X_{1}, X_{2}, X_{3}\right)$ is the coordinate system in the real space.

\subsection{The Homogenized Interface Model}

The acoustic pressure $P(\mathbf{X})$ satisfies the Helmholtz equation outside the inclusions

$$
\left(\Delta+k^{2}\right) P(\mathbf{X})=0,
$$

with $k$ the wavenumber (the time dependance is $e^{-i \omega t}$, with $\omega$ the frequency, and it is omitted in the following). Here, we restricted our study to sound hard inclusions, with Neumann boundary condition on the boundary of the inclusions $\partial_{\mathbf{n}} P=0$, where $\partial_{\mathbf{n}}$ denotes the normal derivative. Also, the wave propagation is associated to a wave source and to a proper radiation condition that we do not need to specify at this point. The inclusions form a periodic arrangement located near the $\left(X_{2}, X_{3}\right)$ plane (with typical thickness $e$ along $X_{1}$, Fig. 14.1(a)). For simplicity, we assume that $h$ is the period of the cell along $X_{2}$ and $X_{3}$ (we could assume two different periods along $X_{2}$ and $X_{3}$ without additional complexity, except in the notations). We inspect the possibility to replace this problem by a simpler one, where the structured film is replaced by an interface associated to jump conditions for the pressure $P$ and its normal derivative, or normal velocity, $\partial_{X_{1}} P$ (the nature of the equivalent interface, in terms of its thickness, will be discussed).

In our acoustic problem, the natural small parameter is $\varepsilon=$ $k h \ll 1$ and to be consistent, we need to write the Helmholtz equation in a dimensionless form, with $\mathbf{x} \equiv k \mathbf{X}$, and $p(\mathbf{x}) \equiv P(\mathbf{X})$. In 


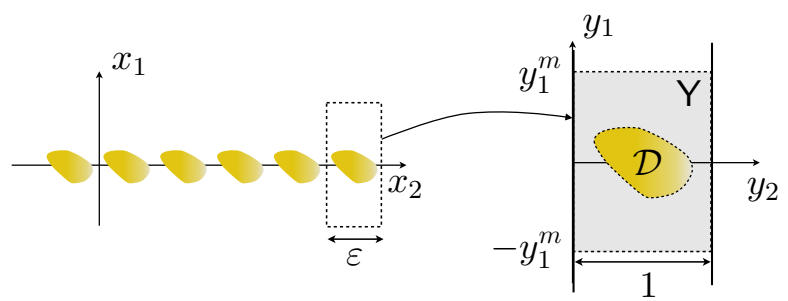

(a)

(b)

Fig. 14.2. (a) Geometry of the structured film in non dimensional coordinates $\mathbf{x}=\left(x_{1}, x_{2}, x_{3}\right)$; (b) inner problem in coordinates $\mathbf{y}=\left(y_{1}, y_{2}, y_{3}\right) . \mathcal{D}$ is the region occupied by the rigid inclusion. We define $\mathrm{Y}=\left(-y_{1}^{m}, y_{1}^{m}\right) \times(0,1)^{2}$, $\mathrm{Y}_{\infty}=\lim _{y_{1}^{m} \rightarrow+\infty} \mathbf{Y}$, and $\mathbf{x}^{\prime}=\left(x_{2}, x_{3}\right), \mathbf{y}^{\prime}=\left(y_{2}, y_{3}\right)$.

$\mathbf{x}=\left(x_{1}, x_{2}, x_{3}\right)$, the period is now $\varepsilon$ and the film thickness is $k e$ (Fig. 14.2(a)); it is assumed that $e$ and $h$ are of the same order of magnitude. We get $\left(\Delta_{\mathbf{x}}+1\right) p(\mathbf{x})=0$, which is written, for convenience using $\mathbf{u}$,

$$
\left\{\begin{array}{l}
\operatorname{div}_{\mathbf{x}} \mathbf{u}(\mathbf{x})+p(\mathbf{x})=0, \quad \text { with } \mathbf{u} \equiv \nabla_{\mathbf{x}} p \\
\mathbf{u} . \mathbf{n}_{\mid \partial \mathcal{D}}=0,
\end{array}\right.
$$

and $\mathbf{u}$ is the acoustic velocity, in dimensionless form.

\subsubsection{The inner/outer expansions and associated matching conditions}

The idea is now to expand the solution with respect to the small parameter $\varepsilon$, namely

$$
\left\{\begin{array}{l}
p=p^{0}(\mathbf{x})+\varepsilon p^{1}(\mathbf{x})+\varepsilon^{2} p^{2}(\mathbf{x})+\ldots, \\
\mathbf{u}=\mathbf{u}^{0}(\mathbf{x})+\varepsilon \mathbf{u}^{1}(\mathbf{x})+\varepsilon^{2} \mathbf{u}^{2}(\mathbf{x})+\ldots
\end{array}\right.
$$

In principle, this expansion can be used in the whole space (see e.g. [Martin and Dalrymple (1988)]). Nevertheless, if the spatial derivatives in Eq. (14.2) make $\varepsilon$ to appear, the resolution may become tricky. Such complications are avoided if a displacement in $\mathbf{x}$ of order unity produces a variation in $p$ of order unity, namely $\partial_{x_{i}} p \sim p$. This 
is ensured in the far field of the film, where $\partial_{X_{i}} P \sim k P \rightarrow \partial_{x_{i}} p \sim p$. The story is different in the near field: there, the film generates evanescent waves whose strongest variations are associated to the smallest scale of the microstructure, say $\partial_{X_{i}} P \sim P / h \rightarrow \partial_{x_{i}} p \sim p / \varepsilon$. Then, the wavefield satisfies pseudo-periodic conditions, for instance along $X_{2}, P\left(X_{1}, X_{2}+h, X_{3}\right)=P\left(X_{1}, X_{2}, X_{3}\right) e^{i k_{2} h}$, with $k_{2}$ the component of the incident wavenumber along $X_{2}$ (the same applies along $\left.X_{3}\right)$. This behavior is associated to low variations of $P\left(\partial_{x_{2}} p \sim\right.$ $p$ ) for non normal incidence $k_{2} \neq 0$. Thus, we have to deal with different scales in the far and near fields, and two scales in the near field.

The presented approach solves this annoying problem with two ingredients: first, a separation of the space into an inner and an outer regions, which correspond to the near and far fields, respectively. In the outer region, the natural coordinates $\mathbf{x} \equiv\left(x_{1}, x_{2}, x_{3}\right)$ are adapted and the expansion, Eq. (14.3), applies. In the inner region, the rapid variations of $p$ and $\mathbf{u}$ are accounted introducing a new system of coordinates $\mathbf{y}=\mathbf{x} / \varepsilon$ such that $\partial_{y_{i}} p \sim p$ for the rapid variations of $p$; next, the slow variations along $x_{2}$ and $x_{3}$ are accounted for by keeping $\mathbf{x}^{\prime} \equiv\left(x_{2}, x_{3}\right)$ as additional coordinates. Note that in the $\mathbf{y}$ coordinates, the cell has a thickness unity (Fig. 14.2(b)). Owing to this modification, the expansions read

$$
\begin{aligned}
& \text { Outer expansion }\left\{\begin{array}{l}
p=p^{0}(\mathbf{x})+\varepsilon p^{1}(\mathbf{x})+\varepsilon^{2} p^{2}(\mathbf{x})+\ldots, \\
\mathbf{u}=\mathbf{u}^{0}(\mathbf{x})+\varepsilon \mathbf{u}^{1}(\mathbf{x})+\varepsilon^{2} \mathbf{u}^{2}(\mathbf{x})+\ldots
\end{array}\right. \\
& \text { Inner expansion }\left\{\begin{array}{l}
p=q^{0}\left(\mathbf{y}, \mathbf{x}^{\prime}\right)+\varepsilon q^{1}\left(\mathbf{y}, \mathbf{x}^{\prime}\right)+\varepsilon^{2} q^{2}\left(\mathbf{y}, \mathbf{x}^{\prime}\right)+\ldots, \\
\mathbf{u}=\mathbf{v}^{0}\left(\mathbf{y}, \mathbf{x}^{\prime}\right)+\varepsilon \mathbf{v}^{1}\left(\mathbf{y}, \mathbf{x}^{\prime}\right)+\varepsilon^{2} \mathbf{v}^{2}\left(\mathbf{y}, \mathbf{x}^{\prime}\right)+\ldots
\end{array}\right.
\end{aligned}
$$

Finally, both regions are connected in some boundary region, where the evanescent field is vanishing at small $x_{1}$ values corresponding to $y_{1}=x_{1} / \varepsilon \rightarrow \pm \infty$. These matching conditions are written using Taylor expansions of $p^{0}\left(x_{1}, \mathbf{x}^{\prime}\right)=p^{0}\left(0, \mathbf{x}^{\prime}\right)+x_{1} \partial_{x_{1}} p^{0}\left(0, \mathbf{x}^{\prime}\right)+\cdots=$ $p^{0}\left(0, \mathbf{x}^{\prime}\right)+\varepsilon y_{1} \partial_{x_{1}} p^{0}\left(0, \mathbf{x}^{\prime}\right)+\ldots$, same for $\mathbf{u}^{0}$, and identifying the terms 
in $\varepsilon^{n}$ in the inner and outer expansions, Eqs. (14.4). We get

$$
\left\{\begin{array}{l}
p^{0}\left(0^{ \pm}, \mathbf{x}^{\prime}\right)=\lim _{y_{1} \rightarrow \pm \infty} q^{0}\left(\mathbf{y}, \mathbf{x}^{\prime}\right) \\
\mathbf{u}^{0}\left(0^{ \pm}, \mathbf{x}^{\prime}\right)=\lim _{y_{1} \rightarrow \pm \infty} \mathbf{v}^{0}\left(\mathbf{y}, \mathbf{x}^{\prime}\right)
\end{array}\right.
$$

at leading order, and

$$
\left\{\begin{array}{l}
p^{1}\left(0^{ \pm}, \mathbf{x}^{\prime}\right)=\lim _{y_{1} \rightarrow \pm \infty}\left[q^{1}\left(\mathbf{y}, \mathbf{x}^{\prime}\right)-y_{1} \frac{\partial p^{0}}{\partial x_{1}}\left(0^{ \pm}, \mathbf{x}^{\prime}\right)\right] \\
\mathbf{u}^{1}\left(0^{ \pm}, \mathbf{x}^{\prime}\right)=\lim _{y_{1} \rightarrow \pm \infty}\left[\mathbf{v}^{1}\left(\mathbf{y}, \mathbf{x}^{\prime}\right)-y_{1} \frac{\partial \mathbf{u}^{0}}{\partial x_{1}}\left(0^{ \pm}, \mathbf{x}^{\prime}\right)\right]
\end{array}\right.
$$

at first order, and fortunately, we do not need to go at higher orders in the expansions. A last subtlety of the interface model is to assume that the functions $q^{n}$ and $\mathbf{v}^{n}$ are periodic with respect to $y_{2}$ and $y_{3}$. Again, this is not meaningless in the present context if we have in mind the condition of pseudo periodicity. This condition is handled by the variables $x_{2}$ and $x_{3}$, for instance $q^{n}\left(\mathbf{y}, x_{2}+\right.$ $\left.\varepsilon, x_{3}\right)=e^{i k_{2} h} q^{n}\left(\mathbf{y}, x_{2}, x_{3}\right)$ (note that the condition of pseudo periodicity applies for the outer solution $\left(p^{n}, \mathbf{u}^{n}\right)$, and from (14.5)-(14.6), it applies for $\left(q^{n}, \mathbf{v}^{n}\right)$ also). If one thinks to the $q^{n}$ in terms of separable functions $q^{n}\left(\mathbf{y}, \mathbf{x}^{\prime}\right)=f(\mathbf{y}) g\left(\mathbf{x}^{\prime}\right)$ (and this will be the case in the quasi-static limit), we recover the form of a Floquet solution, with $g\left(\mathbf{x}^{\prime}\right)=e^{i \mathbf{k}^{\prime} \cdot \mathbf{x}^{\prime} / k}$ and $f$ periodic with respect to $\mathbf{y}^{\prime} \equiv\left(y_{2}, y_{3}\right)$.

Now, the matching conditions, Eqs. (14.5)-(14.6) can be written in an alternative form, that will be used later

$$
\left\{\begin{array}{l}
\llbracket p^{0} \rrbracket=\delta q^{0}, \quad \llbracket p^{1} \rrbracket=\lim _{y_{1}^{m} \rightarrow+\infty}\left[\delta q^{1}\left(y_{1}^{m}\right)-2 y_{1}^{m} \frac{\partial p^{0}}{\partial x_{1}}\left(0, \mathbf{x}^{\prime}\right)\right], \\
\llbracket \mathbf{u}^{0} \rrbracket \cdot \mathbf{e}_{1}=\delta v_{1}^{0}, \llbracket \mathbf{u}^{1} \rrbracket \cdot \mathbf{e}_{1}=\lim _{y_{1}^{m} \rightarrow+\infty}\left[\delta v_{1}^{1}\left(y_{1}^{m}\right)-2 y_{1}^{m} \frac{\partial u_{1}^{0}}{\partial x_{1}}\left(0, \mathbf{x}^{\prime}\right)\right],
\end{array}\right.
$$

(Fig. 14.2), where we have defined

$$
\delta f\left(y_{1}^{m}\right) \equiv f\left(+y_{1}^{m}\right)-f\left(-y_{1}^{m}\right), \quad \text { and } \quad \delta f \equiv \delta f(+\infty) .
$$


In the above expressions, the dependence of $f$ on $\mathbf{y}^{\prime}$ and $\mathbf{x}^{\prime}$ are omitted.

\subsubsection{Equations governing the outer and inner terms at orders 0 and 1}

The equations in the outer and inner problems read, from Eqs. (14.2)

Outer problem: $\operatorname{div}_{\mathbf{x}} \mathbf{u}^{0}+p^{0}=0, \quad \mathbf{u}^{0}=\nabla_{\mathbf{x}} p^{0}$,

$$
\text { Inner problem: }\left\{\begin{array}{l}
\operatorname{div}_{\mathbf{y}} \mathbf{v}^{0}=0, \boldsymbol{\nabla}_{\mathbf{y}} q^{0}=\mathbf{0}, \\
\mathbf{v}^{0}=\nabla_{\mathbf{y}} q^{1}+\nabla_{\mathbf{x}^{\prime}} q^{0}, \\
\operatorname{div}_{\mathbf{x}^{\prime}} \mathbf{v}^{0}+\operatorname{div}_{\mathbf{y}} \mathbf{v}^{1}+q^{0}=0, \\
\mathbf{v}^{0} \cdot \mathbf{n}_{\mid \partial \mathcal{D}}=\mathbf{v}^{1} \cdot \mathbf{n}_{\mid \partial \mathcal{D}}=0 .
\end{array}\right.
$$

For the inner problem, we must add, at orders 0 and 1 , the periodic conditions of $q^{n}$ and $\mathbf{v}^{n}, n=0,1$, with respect to $\mathbf{y}^{\prime}$.

These are the equations that will yield the elementary problems, and, together with the matching conditions, Eqs. (14.5)-(14.6), the jump conditions in the outer problem.

\subsubsection{The jump conditions for the outer problem of order 0}

The first two equations of the inner problem in (14.9) show that $q^{0}$ does not depend on $\mathbf{y}$ and that $\int \mathrm{d} \mathbf{y}^{\prime} v_{1}^{0}\left(y_{1}=-\infty, \mathbf{y}^{\prime}, \mathbf{x}^{\prime}\right)=$ $\int \mathrm{d} \mathbf{y}^{\prime} v_{1}^{0}\left(y_{1}=+\infty, \mathbf{y}^{\prime}, \mathbf{x}^{\prime}\right)$. This latter relation is obtained by integrating $\operatorname{div}_{\mathbf{y}} \mathbf{v}^{0}=0$ over $\mathrm{Y}_{\infty} \backslash \mathcal{D}$ and using the boundary condition $\mathbf{v}^{0} \cdot \mathbf{n}=0$ on $\partial \mathcal{D}$ and the periodicity of $\mathbf{v}^{0}$ with respect to $\mathbf{y}^{\prime}$. It follows from Eqs. (14.5) that (i) $q^{0}\left(\mathbf{x}^{\prime}\right)=p^{0}\left(0^{ \pm}, \mathbf{x}^{\prime}\right)$ and (ii) $\int \mathrm{d} \mathbf{y}^{\prime} v_{1}^{0}\left( \pm \infty, \mathbf{y}^{\prime}, \mathbf{x}^{\prime}\right)=u_{1}^{0}\left(0^{ \pm}, \mathbf{x}^{\prime}\right)$, so that the jump conditions at leading order read

$$
\llbracket p^{0} \rrbracket=\llbracket \mathbf{u}^{0} \rrbracket \cdot \mathbf{e}_{1}=0 .
$$

The structured film is transparent at leading order, with the pressure and the normal velocity being continuous across the interface. In the context of static elasticity, for microcraks or microholes in an elastic 
body, this has been referred as the "the principle of the dressmaker" ([Marigo and Pideri (2011)]): "It is not necessary to sew entirely two pieces of fabrics in order to render invisible their relative opening, it is sufficient to sew them at a great number of points regularly spaced." This is not trivial a priori, and it fact, it is not expected if we have the limiting case of a rigid wall $\ell=h$ in mind, and this will be discussed below. For now, the solution $\left(p^{0}, \mathbf{u}^{0}\right)$ at this leading order is the solution of the Helmholtz equation in the absence of interface, which means that $\left(p^{0}, \mathbf{u}^{0}\right)$ correspond to the pressure and velocity associated to a given incident wave. At this stage, the appropriate radiation condition has to be accounted for to determine the unique solution of the problem at leading order.

To capture the effect of the interface, we need to go at the next order to find the jump conditions.

\subsubsection{The jump conditions for the outer problem of order 1 and the associated elementary problems}

At order 1 , we have to consider a problem on the inner terms $\left(q^{1}, \mathbf{v}^{0}\right)$. To do so, it is convenient to introduce $(q, \mathbf{v})$ defined by

$$
\left\{\begin{array}{l}
q\left(\mathbf{y}, \mathbf{x}^{\prime}\right) \equiv q^{1}\left(\mathbf{y}, \mathbf{x}^{\prime}\right)-y_{1} \frac{\partial p^{0}}{\partial x_{1}}\left(0, \mathbf{x}^{\prime}\right), \\
\mathbf{v}\left(\mathbf{y}, \mathbf{x}^{\prime}\right) \equiv \mathbf{v}^{0}\left(\mathbf{y}, \mathbf{x}^{\prime}\right)-\mathbf{u}^{0}\left(0, \mathbf{x}^{\prime}\right)
\end{array}\right.
$$

From the equations (14.9) in the inner problem, $(q, \mathbf{v})$ satisfy

$$
\left\{\begin{array}{l}
\operatorname{div}_{\mathbf{y}} \mathbf{v}=0, \quad \mathbf{v}=\nabla_{\mathbf{y}} q \\
\lim _{y_{1} \rightarrow \pm \infty} \mathbf{v}=0 \\
\mathbf{v} \cdot \mathbf{n}_{\mid \partial \mathcal{D}}=-\mathbf{u}^{0}\left(0, \mathbf{x}^{\prime}\right) \cdot \mathbf{n}_{\mid \partial \mathcal{D}} \\
q \text { and } \mathbf{v} \cdot \mathbf{n} \text { being } \mathbf{y}^{\prime}-\text { periodic }
\end{array}\right.
$$

where we have used that $q^{0}\left(\mathbf{x}^{\prime}\right)=p^{0}\left(0, \mathbf{x}^{\prime}\right)$ and $\mathbf{u}^{0}=\nabla_{\mathbf{x}} p^{0}$, from the outer problem. For the boundary conditions at $y_{1} \rightarrow \pm \infty$, we 
also used the matching condition, Eq. (14.5), $\mathbf{u}^{0}\left(0, \mathbf{x}^{\prime}\right)=\mathbf{v}^{0}\left(y_{1}=\right.$ $\left.\pm \infty, \mathbf{y}^{\prime}, \mathbf{x}^{\prime}\right)$.

If this problem is solved, the jump conditions can be determined.

Inspecting the form of $\llbracket p^{1} \rrbracket$ in Eqs. (14.7), we see that the jump condition on $p$ is simply determined using the definition of $q$ in (14.11), yielding

$$
\delta q^{1}\left(y_{1}^{m}\right)-2 y_{1}^{m} \frac{\partial p^{0}}{\partial x_{1}}\left(0, \mathbf{x}^{\prime}\right)=\delta q\left(y_{1}^{m}\right),
$$

with the notations (14.8).

For the jump condition on $\mathbf{u}^{1}$, we integrate the third equation of the inner problem in (14.9) over $Y \backslash \mathcal{D}$. This leads to

$$
\delta v_{1}^{1}\left(y_{1}^{m}\right)-\frac{\partial u_{1}^{0}}{\partial x_{1}}\left(0, \mathbf{x}^{\prime}\right)\left(2 y_{1}^{m}-S_{\mathcal{D}}\right)+\frac{\partial}{\partial x_{\alpha}} \int_{\mathbf{Y} \backslash \mathcal{D}} \mathrm{d} \mathbf{y} v_{\alpha}=0,
$$

where $\alpha=(2,3)$, and with $S_{\mathcal{D}}$ the volume of $\mathcal{D}$ in the $\mathbf{y}$ coordinates (we use the summation convention on repeated indices). We have used that (i) $\mathbf{v}^{1}$ is periodic with respect to $\mathbf{y}^{\prime}$, (ii) $q^{0}\left(\mathbf{x}^{\prime}\right)=p^{0}\left(0, \mathbf{x}^{\prime}\right)$ from the previous section and (iii) $\operatorname{div}_{\mathbf{x}^{\prime}} \mathbf{u}^{0}+p^{0}=-\partial_{x_{1}} u_{1}^{0}$ (from the outer problem). Note that $\left(2 y_{1}^{m}-S_{\mathcal{D}}\right)$ is simply the volume of $Y \backslash \mathcal{D}$.

It is now sufficient to take the limit $y_{1}^{m} \rightarrow+\infty$ in Eqs. (14.13)(14.14) and using (14.7) to get

$$
\left\{\begin{array}{l}
\llbracket p^{1} \rrbracket=\delta q, \\
\llbracket \mathbf{u}^{1} \rrbracket \cdot \mathbf{e}_{1}=-\frac{\partial u_{1}^{0}}{\partial x_{1}}\left(0, \mathbf{x}^{\prime}\right) S_{\mathcal{D}}-\frac{\partial}{\partial x_{\alpha}} \int_{\mathbf{Y}_{\infty} \backslash \mathcal{D}} \mathrm{d} \mathbf{y} v_{\alpha}\left(\mathbf{y}, \mathbf{x}^{\prime}\right),
\end{array}\right.
$$

with $Y_{\infty}=(-\infty, \infty) \times(0,1)^{2}$.

As written above, the jump conditions are not satisfactory in the sense that both $q$ and $v_{\alpha}$ depend on the value of $\mathbf{u}^{0}$ on $\partial \mathcal{D}$ (in (14.12)). However, it is possible to split the problem (14.12) on $(\mathbf{v}, q)$ into simple problems on $q^{(i)}(\mathbf{y}), i=1,2,3$, called elementary problems. These problems are "elementary" in the sense that they can be solved once and for all and this is because these problems are 
independent of $\mathbf{x}^{\prime}$ and of the value of $\mathbf{u}^{0}$ on $\partial \mathcal{D}$. Specifically, $(q, \mathbf{v})$ can be decomposed into

$$
\left\{\begin{array}{l}
q\left(\mathbf{y}, \mathbf{x}^{\prime}\right)=\frac{\partial p^{0}}{\partial x_{i}}\left(0, \mathbf{x}^{\prime}\right) q^{(i)}(\mathbf{y})+\hat{q}\left(\mathbf{x}^{\prime}\right) \\
\mathbf{v}\left(\mathbf{y}, \mathbf{x}^{\prime}\right)=\frac{\partial p^{0}}{\partial x_{i}}\left(0, \mathbf{x}^{\prime}\right) \nabla q^{(i)}(\mathbf{y})
\end{array}\right.
$$

with $q^{(i)}(\mathbf{y})$ satisfying

$$
\text { the elementary problems: }\left\{\begin{array}{l}
\Delta q^{(i)}=0, \\
\boldsymbol{\nabla}\left[q^{(i)}+y_{i}\right] . \mathbf{n}=0, \text { on } \partial \mathcal{D}, \\
\lim _{y_{1} \rightarrow \pm \infty} \nabla q^{(i)}=0 \\
q^{(i)} \text { being } \mathbf{y}^{\prime}-\text { periodic. }
\end{array}\right.
$$

It is easy to see that such decomposition ensures that $(q, \mathbf{v})$ is solution of (14.12) (using $\mathbf{u}^{0}=\boldsymbol{\nabla}_{\mathbf{x}} p^{0}$ ). Note that $\hat{q}\left(\mathbf{x}^{\prime}\right)$ has been introduced since $q\left(\mathbf{y}, \mathbf{x}^{\prime}\right)$ in (14.12) is defined up to a $\mathbf{x}^{\prime}$-dependent field, but its determination is not needed.

We can now express $\delta q$ and $v_{\alpha}$ in (14.15) in terms of the elementary solutions $q^{(i)}$

$$
\left\{\begin{array}{l}
\delta q=\frac{\partial p^{0}}{\partial x_{i}}\left(0, \mathbf{x}^{\prime}\right) \delta q^{(i)} \\
v_{\alpha}\left(\mathbf{y}, \mathbf{x}^{\prime}\right)=u_{i}^{0}\left(0, \mathbf{x}^{\prime}\right) \frac{\partial q^{(i)}}{\partial y_{\alpha}}(\mathbf{y})
\end{array}\right.
$$

and the jump conditions (14.15) become

$$
\left\{\begin{array}{l}
\llbracket p^{1} \rrbracket=\frac{\partial p^{0}}{\partial x_{i}}\left(0, \mathbf{x}^{\prime}\right) \delta q^{(i)}, \\
\llbracket \mathbf{u}^{1} \rrbracket \cdot \mathbf{e}_{1}=-\frac{\partial u_{1}^{0}}{\partial x_{1}}\left(0, \mathbf{x}^{\prime}\right) S_{\mathcal{D}}-\frac{\partial u_{i}^{0}}{\partial x_{\alpha}}\left(0, \mathbf{x}^{\prime}\right) \int_{Y_{\infty} \backslash \mathcal{D}} \mathrm{d} \mathbf{y} \frac{\partial q^{(i)}}{\partial y_{\alpha}}(\mathbf{y}),
\end{array}\right.
$$


which can be written introducing the parameters $\mathbf{b}$ and $\mathbf{c}$

$$
\left\{\begin{array}{l}
\llbracket p^{1} \rrbracket=b_{i} \frac{\partial p^{0}}{\partial x_{i}}\left(0, \mathbf{x}^{\prime}\right), \\
\llbracket \mathbf{u}^{1} \rrbracket \cdot \mathbf{e}_{1}=c_{i j} \frac{\partial u_{i}^{0}}{\partial x_{j}}\left(0, \mathbf{x}^{\prime}\right),
\end{array}\right.
$$

with $b_{i}=\delta q^{(i)}$ and

$$
\left\{\begin{array}{l}
\mathrm{c}_{11}=-S_{\mathcal{D}}, \mathrm{c}_{1 \beta}=-\int_{\mathrm{Y}_{\infty} \backslash \mathcal{D}} \mathrm{d} \mathbf{y} \frac{\partial q^{(1)}}{\partial y_{\beta}}, \\
\mathrm{c}_{\alpha 1}=0, \quad \mathrm{c}_{\alpha \beta}=-\int_{\mathrm{Y}_{\infty} \backslash \mathcal{D}} \mathrm{d} \mathbf{y} \frac{\partial q^{(\alpha)}}{\partial y_{\beta}},
\end{array}\right.
$$

where $\alpha, \beta$ take the values 2 and 3 .

It is essential that the elementary problems, Eqs. (14.17), do not depend on the incident wave (as the former problem Eqs. (14.12), does, through $\mathbf{u}^{0}$ in the boundary condition). This ensures that the parameters $\mathbf{b}$ and $\mathbf{c}$ are characteristic of the interface independently of the particular scattering problem that will be considered.

\subsubsection{Construction of the up to order 1 effective problem}

The final up to order 1 effective problem requires two additional steps. First, we have obtained jump conditions at order 0 and at order 1. Strictly, an iterative resolution of the problem is possible which would involve first the resolution of the order 0 outer problem (which does not see the structured layer) yielding $\left(\mathbf{u}^{0}, p^{0}\right)$, and then the resolution of the order 1 outer problem associated to the jump conditions (14.20) (the effective parameters entering in (14.20) being known after the elementary problems (14.17) have been solved). However, it is stressed in [David et al. (2012)] that such procedure is not suitable in practice, notably if we have numerical resolutions in mind, and rather, a unique outer problem involving $p^{0}+\varepsilon p^{1}$ (and $u^{0}+\varepsilon u^{1}$ ) has to be considered. To do so, we use $f=f^{0}+\varepsilon f^{1}+O\left(\varepsilon^{2}\right)$ and $\llbracket f \rrbracket=\varepsilon \llbracket f^{1} \rrbracket+O\left(\varepsilon^{2}\right)$ for any field $f$ with $\llbracket f^{0} \rrbracket=0$. From Eqs. (14.10) 
and (14.20), we get

$$
\left\{\begin{array}{l}
\llbracket p \rrbracket=\varepsilon \frac{b_{i}}{2}\left[\frac{\partial p}{\partial x_{i}}\left(0^{-}, \mathbf{x}^{\prime}\right)+\frac{\partial p}{\partial x_{i}}\left(0^{+}, \mathbf{x}^{\prime}\right)\right]+O\left(\varepsilon^{2}\right), \\
\llbracket \mathbf{u} \rrbracket \cdot \mathbf{e}_{1}=\varepsilon \frac{\mathbf{c}_{i j}}{2}\left[\frac{\partial u_{i}}{\partial x_{j}}\left(0^{-}, \mathbf{x}^{\prime}\right)+\frac{\partial u_{i}}{\partial x_{j}}\left(0^{+}, \mathbf{x}^{\prime}\right)\right]+O\left(\varepsilon^{2}\right),
\end{array}\right.
$$

where we have introduced for fields being discontinuous at $x_{1}=0$ the mean value of their limits $x_{1} \rightarrow 0^{ \pm}$.

Next, the above conditions will be written in an alternative form, equivalent at order $O\left(\varepsilon^{2}\right)$. Loosely speaking, the new jump conditions have to restore the initial thickness of the structured layer, and this is required following the intuitive argument that the equivalent interface has to support the acoustic energy in the actual layer in order to ensure that it supports a positive energy, see [Marigo and Maurel (2016)] for more details. In [Abdelmoula et al. (1998)], this is done by considering that the problem is written in a domain of fixed size in which the film of size $\varepsilon$ has been inserted, resulting in a shift of the boundaries. In [Delourme (2010); Delourme et al. (2012)], this is done to overcome troubles in the homogenized problem due to the signs of the $\left(b_{i}, c_{i j}\right)$, specifically some of these parameters have to be positive or negative to ensure existence and uniqueness of variational solutions in the homogenized problem. In this latter case, a family of equivalent jump conditions are proposed, which correspond to interfaces "enlarged" of an arbitrary thickness; here, we consider a shift of $e$. A shift of $e$ in the real space corresponds to a shift of $k e$ in the $\mathbf{x}$-space and we assume that $e / h=O(1)$, from which $k e=\varepsilon e / h=O(\varepsilon)$. To do so, we use the Taylor expansion of $p^{n}$, $n=0,1$,

$$
\left\{\begin{array}{l}
p^{0}\left( \pm \frac{\varepsilon e}{2 h}, \mathbf{x}^{\prime}\right)=p^{0}\left(0, \mathbf{x}^{\prime}\right) \pm \frac{\varepsilon e}{2 h} \frac{\partial p^{0}}{\partial x_{1}}\left( \pm \frac{\varepsilon e}{2 h}, \mathbf{x}^{\prime}\right)+O\left(\varepsilon^{2}\right) \\
p^{1}\left( \pm \frac{\varepsilon e}{2 h}, \mathbf{x}^{\prime}\right)=p^{1}\left(0^{ \pm}, \mathbf{x}^{\prime}\right)+O(\varepsilon)
\end{array}\right.
$$


to get

$$
\begin{aligned}
p\left(\frac{\varepsilon e}{2 h}, \mathbf{x}^{\prime}\right)-p\left(-\frac{\varepsilon e}{2 h}, \mathbf{x}^{\prime}\right)= & \frac{\varepsilon e}{2 h}\left[\frac{\partial p^{0}}{\partial x_{1}}\left(-\frac{\varepsilon e}{2 h}, \mathbf{x}^{\prime}\right)+\frac{\partial p^{0}}{\partial x_{1}}\left(\frac{\varepsilon e}{2 h}, \mathbf{x}^{\prime}\right)\right] \\
& +\varepsilon \llbracket p^{1} \rrbracket+O\left(\varepsilon^{2}\right) .
\end{aligned}
$$

Finally, defining for any $f$, the mean value of $f$ across the interface of thickness $k e$

$$
\bar{f}=\frac{1}{2}\left[f\left(-\frac{\varepsilon e}{2 h}, \mathbf{x}^{\prime}\right)+f\left(\frac{\varepsilon e}{2 h}, \mathbf{x}^{\prime}\right)\right],
$$

and coming back to (14.26), the new jump conditions read

$$
\left\{\begin{array}{l}
\llbracket p \rrbracket_{e}=\varepsilon\left(b_{i}+\frac{e}{h} \delta_{i 1}\right) \overline{\frac{\partial p}{\partial x_{i}}}+O\left(\varepsilon^{2}\right), \\
\llbracket \mathbf{u} \rrbracket_{e} \cdot \mathbf{e}_{1}=\varepsilon\left(\mathrm{c}_{i j}+\frac{e}{h} \delta_{i 1} \delta_{j 1}\right) \overline{\frac{\partial u_{i}}{\partial x_{j}}}+O\left(\varepsilon^{2}\right) .
\end{array}\right.
$$

Now, the homogenized model is written in the real space for the pressure field $P(\mathbf{X})$ (Fig. 14.3): $P$ satisfies the Helmholtz equation $\Delta P+k^{2} P=0$ except in $-e / 2<X_{1}<e / 2$ (this region is disregarded) and it experiences jump conditions which link its values at $X_{1}=$ $\pm e / 2$, with $\llbracket P \rrbracket_{e} \equiv P\left(e / 2, \mathbf{X}^{\prime}\right)-\left(-e / 2, \mathbf{X}^{\prime}\right)$, to the mean values of its spatial derivatives $\overline{\partial_{X_{i}} P} \equiv \frac{1}{2}\left[\partial_{X_{i}} P\left(-e / 2, \mathbf{X}^{\prime}\right)+\partial_{X_{i}} P\left(e / 2, \mathbf{X}^{\prime}\right)\right]$

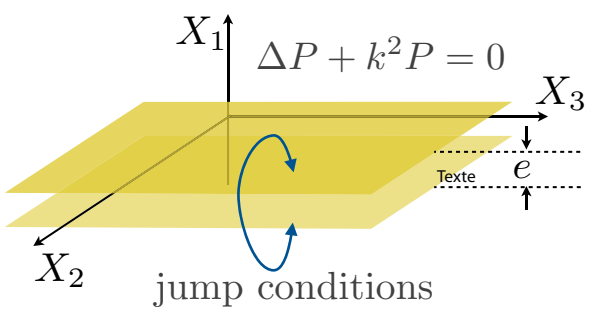

Fig. 14.3. In the homogenized problem, the Helmholtz equation applies in the whole-considered-space except in the region $-e / 2<X_{1}<e / 2$ which is disregarded; the jump conditions (14.27) apply between $X_{1}=-e / 2$ and $e / 2$. 
(the same for $\partial_{X_{1}} P$ ):

$$
\text { Jump conditions }\left\{\begin{array}{l}
\llbracket P \rrbracket_{e}=h B_{i} \frac{\overline{\partial P}}{\partial X_{i}}, \\
\llbracket \frac{\partial P}{\partial X_{1}} \rrbracket_{e}=h \mathrm{C}_{i j} \frac{\partial^{2} P}{\partial X_{i} \partial X_{j}},
\end{array}\right.
$$

with

$$
\begin{cases}B_{1}=\frac{e}{h}+\delta q^{(1)}, & B_{\alpha}=\delta q^{(\alpha)}, \\ \mathrm{C}_{11}=\frac{e}{h}-S_{\mathcal{D}}, & \mathrm{C}_{1 \beta}=-\int_{\mathrm{Y}_{\infty} \backslash \mathcal{D}} \mathrm{d} \mathbf{y} \frac{\partial q^{(1)}}{\partial y_{\beta}}, \\ \mathrm{C}_{\alpha 1}=0, & \mathrm{C}_{\alpha \beta}=-\int_{\mathrm{Y}_{\infty} \backslash \mathcal{D}} \mathrm{d} \mathbf{y} \frac{\partial q^{(\alpha)}}{\partial y_{\beta}},\end{cases}
$$

where $\alpha, \beta$ take the values 2 and 3 .

\subsubsection{Remarks}

As previously said, it is essential that the elementary problems, Eqs. (14.17), do not depend on the incident wave. This ensures that the interface parameters are characteristic of the structured film only. However, one can think to introduce a dependance on the frequency in the elementary problems, as it has been done for classical homogenization (high frequency homogenization or resonant homogenization) [Bouchitté and Felbacq (2004); Felbacq and Bouchitté (2005); Craster et al. (2011); Antonakakis et al. (2013)]. For acoustic waves, this could be done for metafilms containing long hard scatterers wrapped in the unit cell, thus allowing for the resonance of the enrolled element. For elastic or electromagnetic waves, this could be done for metafilms containing inclusions with a contrast in its material properties such that the wavelength inside the inclusions is of the same order than the size of the inclusions.

The following remarks can be done on the elementary problems, Eqs. (14.17) 
- The problem for $i=1$ is nothing but the problem of a uniform potential flow past a rigid obstacle in a duct. With velocity potential $\varphi(\mathbf{y})=q^{(1)}+y_{1}$, we get $\Delta \varphi=0, \partial_{n} \varphi_{\mid \partial \mathcal{D}}=0$ and $\lim _{y_{1} \rightarrow \pm \infty} \varphi=y_{1}+b^{ \pm}$. The coefficient $b_{1}=b^{+}-b^{-}$is called the blockage coefficient and it has been calculated for several shapes of obstacles (see [Martin and Dalrymple (1988)] and references therein). This coefficient can be non zero even for zero surface obstacle $S_{\mathcal{D}}=0$, reducing to a plate of length $\ell$.

- For inclusion shape being symmetric with respect to $y_{2}, q^{(1)}$ is symmetric also. It results that $\int_{Y \backslash \mathcal{D}} \mathrm{d} \mathbf{y} \partial_{y_{\alpha}} q^{(1)}=0$.

- The cell problem for $i=2,3$ is needed only for oblique incidence of the wave. Besides, for inclusion shape being symmetric with respect to $y_{2}, q^{(\alpha)}, \alpha=2,3$ are antisymmetric; in this case, $\delta q^{(\alpha)}=0$.

Finally, we have said that the present derivation of the jump conditions follows from previous works [Marigo and Pideri (2011); David et al. (2012)] in the static case $(k=0)$. For the sake of completeness, we report in the Appendix 14.5 a short discussion on the links with this work.

\subsection{The Case of Sound Hard Rectangular Inclusions in $2 \mathrm{D}$}

In the following, we consider a 2 dimensional array made of rectangular inclusions infinite along $X_{3}$ (otherwise of size $\ell$ along $X_{2}$ and thickness e along $X_{1}$ ). This inclusion shape being symmetric with respect to $y_{2}$, the preceding remarks on the elementary problems apply and the jump conditions (14.27) simplify to

$$
\text { Jump conditions }\left\{\begin{array}{l}
\llbracket P \rrbracket_{e}=h B \overline{\frac{\partial P}{\partial X_{1}}}, \\
\llbracket \frac{\partial P}{\partial X_{1}} \rrbracket_{e}=h S \frac{\overline{\partial^{2} P}}{\partial X_{1}^{2}}+h C \frac{\overline{\partial^{2} P}}{\partial X_{2}^{2}},
\end{array}\right.
$$


with $S \equiv\left(e / h-S_{\mathcal{D}}\right), B \equiv e / h+\delta q^{(1)}$ and $C \equiv-\int_{Y_{\infty} \backslash \mathcal{D}} \mathrm{d} \mathbf{y} \partial_{y_{2}} q^{(2)}$. As previously, $\bar{f}$ means the mean value of $f$ at $X_{1}= \pm e / 2$.

\subsubsection{The interface parameters}

An obvious interface parameter is $S=e / h(1-\ell / h)$. Next, $(B, C)$ can be calculated by solving numerically the elementary problems, Eqs. (14.17). A simple procedure to calculate $(B, C)$ (based on mode matching) and the associated scripts are provided in the Appendice 14.6. Alternatively, we can use approximate but explicit expressions. An estimate of $C$ is, as a rule of thumb,

$$
\begin{cases}C \simeq e\left(1-\frac{\ell}{h}\right)-\frac{\pi}{8}\left(1-\frac{\ell}{h}\right)^{2}, & \text { if this leads to a positive value, } \\ C \simeq 0, & \text { otherwise }\end{cases}
$$

which means that $C$ (which is always positive) can be neglected for small $e / h$ values. A more precise estimate of $B$ can be found in [Flagg and Newman (1971)] (see also [Suh et al. (2011)]). It is written as the sum of two terms, one of which does not vanish for $e=0$ (blockage coefficient of a flat plate)

$$
\left\{\begin{array}{l}
B=\frac{e}{h-\ell}+B_{0}, \\
B_{0}=\frac{2}{\pi} \log \left(\frac{d}{2}+\frac{1}{2 d}\right), \quad \text { with } d \equiv \tan \left(\frac{\pi(h-\ell)}{4 h}\right) .
\end{array}\right.
$$

In fact, Ref. [Flagg and Newman (1971)] provides an approximation of $B_{0} \simeq \frac{2}{\pi}\left[1-\log (4(1-\ell / h))+\frac{1}{3}(1-\ell / h)^{2}+\frac{281}{180}(1-\ell / h)\right]$ valid for $\ell / h$ close to 1 . The expression of $B_{0}$ in Eq. (14.31) above can be found in [Morse and Ingard (1968)] (the calculation is done for flat plates using techniques of complex variables). The parameters $(B, C)$ are reported in Fig. 14.4. 

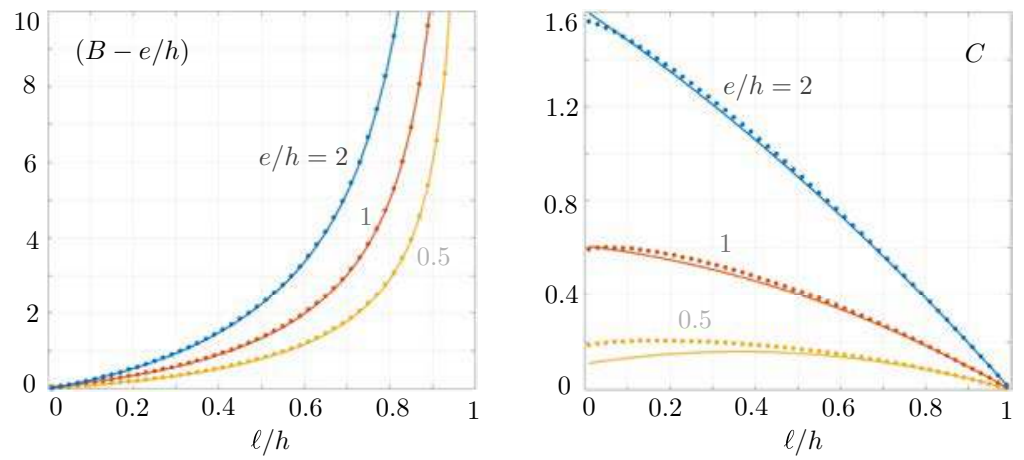

Fig. 14.4. Interface parameters $B$ and $C$ computed numerically by solving the elementary problems (14.17) (symbols), see Appendix 14.6. (a) $(B-e / h)$ as a function of $\ell / h$ for $e / h=0.5,1$ and 2 ; plain lines show the values given by (14.31). Same representation for $C$, with plain lines given by (14.30).

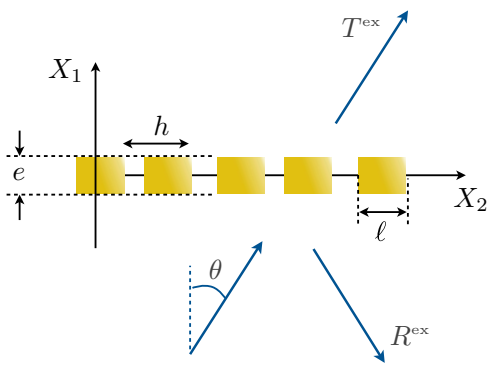

(a)

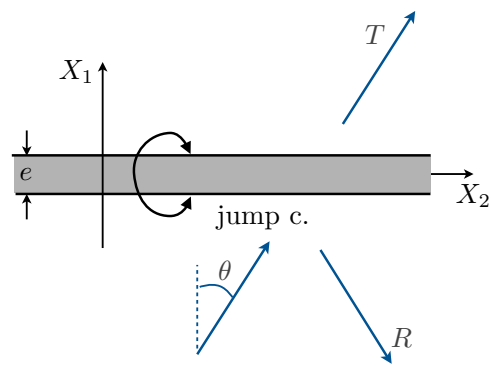

(b)

Fig. 14.5. Scattering of a plane wave at oblique incidence $\theta$ (a) in the real problem, $\left(R^{\mathrm{ex}}, T^{\mathrm{ex}}\right)$ refer to the reflection and transmission coefficients of the incident plane wave, characteristic of the propagating waves and an evanescent near field is generated in the vicinity of the film, (b) in the homogenized problem, the jump conditions (14.29) do not produce evanescent field and $(R, T)$ refers simply to the reflection and transmission coefficients of the incident wave.

\subsubsection{Validity of the interface model - The scattering of an incident plane wave}

We consider the problem of the scattering of an incident plane wave at oblique incidence $\theta$ on the structured film (Fig. 14.5). When replaced by the equivalent interface associated to the jump conditions Eqs. (14.29), the problem reduces to a one dimensional problem 
whose solution reads

$$
\left\{\begin{array}{l}
P\left(X_{1}<-e / 2, X_{2}\right)=e^{\mathrm{i} k \sin \theta X_{2}}\left[e^{\mathrm{i} k \cos \theta\left(X_{1}-e / 2\right)}+R e^{-\mathrm{i} k \cos \theta\left(X_{1}-e / 2\right)}\right], \\
P\left(X_{1}>e / 2, X_{2}\right)=e^{\mathrm{i} k \sin \theta X_{2}} T e^{\mathrm{i} k \cos \theta\left(X_{1}+e / 2\right)},
\end{array}\right.
$$

with

$$
\left\{\begin{array}{l}
R=\mathrm{i} \frac{a-b}{(1-\mathrm{i} a)(1-\mathrm{i} b)}, \quad T=\frac{1+a b}{(1-\mathrm{i} a)(1-\mathrm{i} b)}, \\
\text { with } a \equiv \frac{k h}{2} \cos \theta\left(S+C \tan ^{2} \theta\right), b \equiv \frac{k h}{2} \cos \theta B .
\end{array}\right.
$$

Note that for $\ell \rightarrow h$, we have $B \rightarrow+\infty$ (thus $b \rightarrow+\infty$ ) and $C \rightarrow 0$. We obtain $R \rightarrow-1 /(1-\mathrm{i} a)$ and $T \rightarrow \mathrm{i} a /(1-\mathrm{i} a)$, with $a \propto S \neq 0$ except for a flat array $e=0$. Obviously, we would expect $R=1, T=0$ in this case since the array becomes a sound hard wall. It is a classical problem in homogenization theories when a new small parameter is introduced, here $\varepsilon^{\prime}=1-\ell / h$. In the present case, the problem $\varepsilon^{\prime} \rightarrow 0$ appears in the jump conditions, Eq. (14.26); it has been assumed that $\varepsilon B \ll 1$ (as it has been assumed that $\varepsilon C \ll 1$ and $\varepsilon S \ll 1)$. Inspecting $B$ in Eq. (14.31), we see that this assumption can fail when $(h-\ell) \rightarrow 0$, depending on how fast $\varepsilon$ and $\varepsilon^{\prime}$ go to zero: (i) if $e \neq 0$, it fails if $\varepsilon / \varepsilon^{\prime} \rightarrow \infty$ and (ii) if $e=0$, it fails if $\varepsilon \log \varepsilon^{\prime} \rightarrow \infty$. This latter limit corresponds to the one established in [Sanchez-Hubert and Sanchez-Palencia (1982)] for perforated thin plate.

In the actual problem, the incident wave generates an evanescent field in the vicinity of the structured film, which may have a strong influence on the reflection and transmission of the plane wave. Below, we inspect the validity of our homogenized solution, namely its ability to incorporate the effect of the evanescent field in the interface parameters.

To begin with, we report in Figs. 14.6 and 14.7 the pressure fields for a structured interface of thickness $e$, with $k e=0.5$ and 1 and, in the unit cell, different aspect ratio $\ell / h$. For the considered geometries, the interface parameters are given in the table below. The notation $\mathrm{X}[\mathrm{Y}]$ is used, with $\mathrm{X}$ the value of $B$ or $C$ given by the estimates 

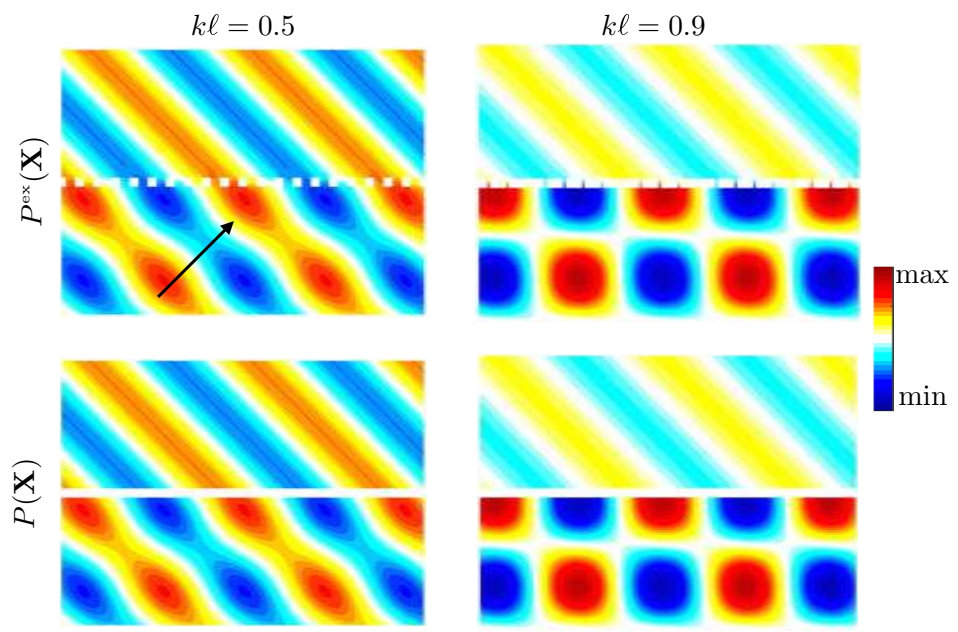

Fig. 14.6. Wavefields in the $\left(X_{1}, X_{2}\right)$ plane for $k e=0.5, k h=1$, and $k \ell=0.5$ or 0.9 (a) $P^{\text {ex }}(\mathbf{X})$ and (b) $P(\mathbf{X})$ coming from the interface model, Eqs. (14.32)(14.33). The white arrow indicates the wavenumber of the incident plane wave.

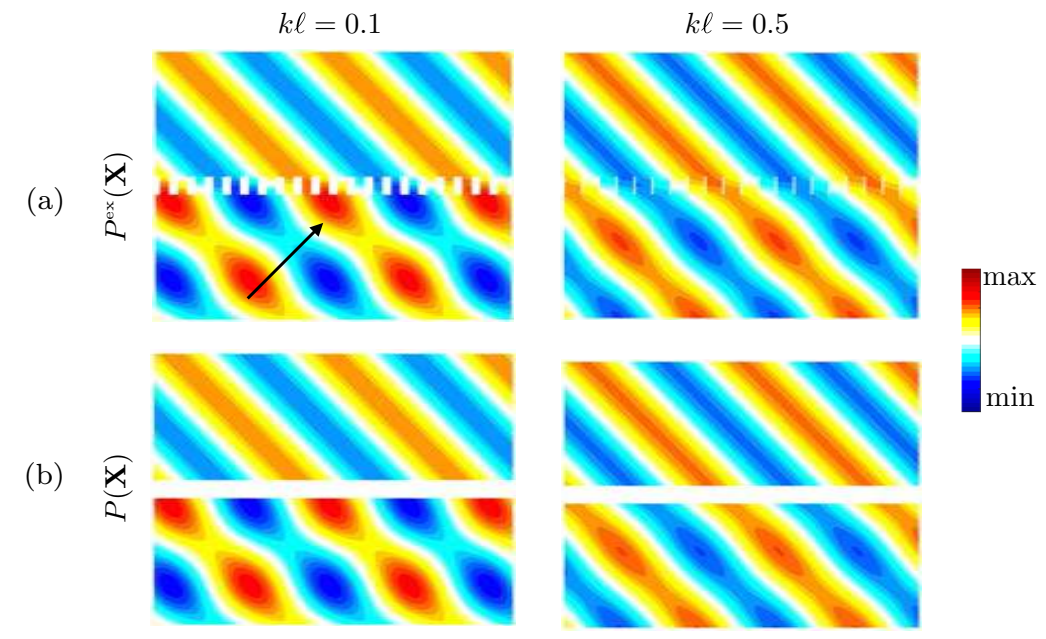

Fig. 14.7. Same representation as in Fig. 14.6 for $k e=1 k h=1$, and $k \ell=0.1$ or 0.5 . 
(14.30)-(14.31), and Y the value obtained by solving the associated elementary problem (see Fig. 14.4 and Appendix 14.6).

\begin{tabular}{|l|c|c|}
\hline$e / h=0.5$ & $\ell / h=0.5$ & $\ell / h=0.9$ \\
\hline$B$ & $1.22[1.25]$ & $6.18[6.22]$ \\
$C$ & $0.15[0.17]$ & $0.05[0.05]$ \\
\hline
\end{tabular}

\begin{tabular}{|l|c|c|}
\hline$e / h=1$ & $\ell / h=0.1$ & $\ell / h=0.5$ \\
\hline$B$ & $1.12[1.12]$ & $2.22[2.25]$ \\
$C$ & $0.58[0.60]$ & $0.40[0.41]$ \\
\hline
\end{tabular}

The field $P^{\mathrm{ex}}(\mathbf{X})$ refers to the solution of the actual problem calculated numerically (a multimodal method is used, see [Maurel et al. (2014)]) and $P(\mathbf{X})$ refers to the prediction coming from the interface model, Eq. (14.32), with Eqs. (14.33). We have considered $\theta=\pi / 4$, $k h=1$. This $\varepsilon=k h=1$ value is obviously a limiting case for the validity of any homogenization model but the fields $P^{\text {ex }}$ and $P$ are found in good agreement, with $\left\|P-P^{\mathrm{ex}}\right\| /\left\|P^{\mathrm{ex}}\right\| \sim 1 \%$ for $k e=0.5$ (in both cases reported in Figs. 14.6) and $\left\|P-P^{\mathrm{ex}}\right\| /\left\|P^{\mathrm{ex}}\right\| \sim 5 \%$ for $k e=1$ (in both cases reported in Figs. 14.7); $\|$.$\| is the norm L_{2}$, considering the field in $\left|X_{1}\right|>e / 2$.

To go further, we inspect the variations of the reflection coefficient as a function of $k$ and $e$ (say for a fixed $h$ value). This is done by comparing $R$ in Eq. (14.33) (blue dotted lines in Figs. 14.8) with $R^{\text {ex }}$ computed numerically (plain orange lines). We also report the behavior of $\tilde{R}$ (grey dotted lines) given by the classical homogenization of layered media (see Appendix 14.7.1). In this approach, the propagation inside the structured layer is explicitly accounted for (while the boundary layer effects are disregarded) and the structured film is replaced by an equivalent film of thickness $e$ filled with an homogeneous anisotropic medium. Classical homogenization has been shown efficient to predict the extraordinary optical transmission at the Brewster angle [Maurel et al. (2013); Akarid et al. (2014)] and the behavior of "spoof plasmons", which are the waves guided 


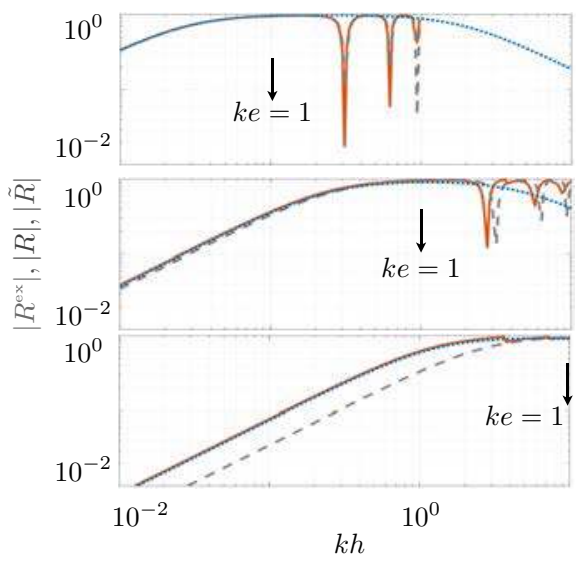

(a)



(b)

Fig. 14.8. Variations of the reflection coefficients $R^{\text {ex }}$ calculated numerically (orange plain lines), $R$ given by the interface model, Eq. (14.33) (blue dotted lines), and $\tilde{R}$ given by classical homogenization, Eq. (14.34) (grey dotted lines). (a) as a function of $k h$ for $e / h=10,1,10^{-1}$ (from the top to the bottom), and (b) as a function of $e / h$ for $k h=10^{-2}, 10^{-1}, 1$ (from the top to the bottom). In all plots, $\ell=0.9 h$ and $\theta=\pi / 4$.

along the boundaries of a thick structured array [Garcia-Vidal et al. (2005); Mercier et al. (2015)]. It has also been applied for the design of a structure producing a rainbow type effect [Zhu et al. (2013)] (see the supplementary material in this reference). In the case of sound hard layers, this leads to a reflection coefficient $\tilde{R}$

$$
\tilde{R}=\frac{\left(c^{2}-1\right) e^{\mathrm{i} k e \cos \theta / 2}}{[c-\mathrm{i} \tan (k e / 2)][c+\mathrm{i} / \tan (k e / 2)]},
$$

where $c=h \cos \theta /(h-\ell)$ (the derivation is given in the Appendix 14.7.1).

From Figs. 14.8, the ranges of validity of both homogenizations can be defined: the interface homogenization is valid for $k e, k h<1$, while the classical homogenization is valid for $e / h>1$, specifically $k e>1>k h$ (inspecting higher values of $k h$ would reveal that both homogenizations fail for $k h>1$, as expected). The error in $\tilde{R}$ for 
small $e / h$ is attributable to the behavior of $B$ when $k e \rightarrow 0$, namely

$$
\lim _{e \rightarrow 0} R \simeq-\mathrm{i} \frac{k h}{2} \cos \theta B_{0},
$$

and $B_{0} \neq 0$ while $\tilde{R} \rightarrow 0$ (a discussion on the jump conditions obtained from the classical homogenization in the limit $k e \rightarrow 0$ is presented in the Appendix 14.7.2). Thus, classical homogenization underestimates the scattering properties of thin films.

\subsubsection{Application to the wave propagation in a guide obstructed by a hard perforated screen}

In addition to yield explicit solutions of several scattering problems, homogenization approaches are of particular interest if we have numerical resolutions in mind. This is particularly true if the problem under consideration involves very different scales between the largest one and the smallest one, and this is what we exemplify in this section. We consider the high frequency propagation of an acoustic wave in a rigid guide of height $H$, with $k H=100$ (Fig. 14.9). The guide is obstructed, from wall to wall, by a rigid perforated screen with $h=H / 100$ and $e=H / 2000$ (thus, $k h=1$ and $k e=0.05$ ). Thus, the smallest scale is $e$ and the largest scale is $H$, with $H=2000 e$ and this is demanding in terms of any computational method.

In general, the solution of guided wave problems can be expanded in modes, associated to transverse functions $\varphi_{n}\left(X_{2}\right)$ with vanishing

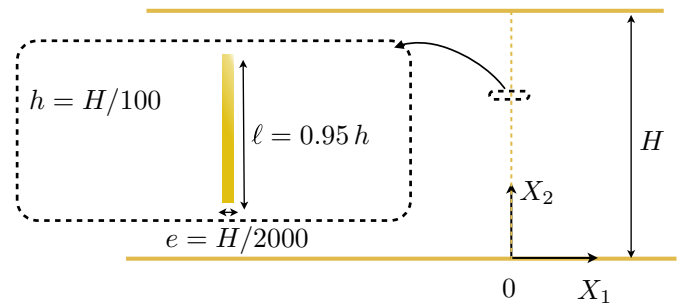

Fig. 14.9. Configuration of the rigid waveguide obstructed by a perforated screen at $-e / 2<X_{1}<e / 2$. The screen has a periodic spacing $h=H / 100$ and thickness $e=H / 2000$. The height of the rigid material in the screen is $\ell / h=0.95$. The guide is studied in the high frequency regime, with $k H=100$. 
first derivative at $X_{2}=0, H$, namely

$$
\left\{\begin{array}{l}
P\left(X_{1}, X_{2}\right)=\sum_{n=0}^{N} P_{n}\left(X_{1}\right) \varphi_{n}\left(X_{2}\right), \\
\varphi_{n}\left(X_{2}\right)=\sqrt{2-\delta_{n 0}} \cos \left(\frac{n \pi X_{2}}{H}\right),
\end{array}\right.
$$

(the $\sqrt{2}$ stands for proper normalization of the transverse functions). At this stage, $P$ stands for the solution of the actual problem or for the solution of the homogenized one. For $N \rightarrow+\infty,(14.36)$ is the modal expansion of the solution, the $P_{n}$ being the projections of $P$ on the basis $\varphi_{n}$; in the numerics, a finite and sufficiently large $N$-value is used within a multimodal approach which accounts for the mode coupling due the presence of the screen, see [Maurel and Mercier (2012); Maurel et al. (2014)].

However, in the homogenized problem, no coupling is expected. This is because the jump conditions, Eqs. (14.29), apply for each mode owing to the orthogonality of the transverse functions, and they take the form

$$
\left\{\begin{array}{l}
\llbracket P_{n} \rrbracket=h B \overline{P_{n}^{\prime}}, \\
\llbracket P_{n}^{\prime} \rrbracket=h S \overline{P_{n}^{\prime \prime}}-h C\left(\frac{n \pi}{H}\right)^{2} \overline{P_{n}},
\end{array}\right.
$$

where we used that $\varphi_{n}^{\prime \prime}=-(n \pi / H)^{2} \varphi_{n}$. Next, in the parts of the waveguide free of scatterer, each mode satisfies $P_{n}^{\prime \prime}+k_{n}^{2} P_{n}=0$, with

$$
k_{n} \equiv \sqrt{k^{2}-\left(\frac{n \pi}{H}\right)^{2}}
$$

the component of the wavenumber along $X_{1}$. Except at the interface $-e / 2<X_{1}<e / 2$, this is the case and we can write for each $P_{n}$ $P_{n}\left(X_{1}\right)= \begin{cases}P_{n}^{\mathrm{inc}}\left[e^{\mathrm{i} k_{n}\left(X_{1}+e / 2\right)}+r_{n} e^{-\mathrm{i} k_{n}\left(X_{1}+e / 2\right)}\right], & \text { for } X_{1}<-e / 2, \\ P_{n}^{\mathrm{inc}} t_{n} e^{\mathrm{i} k_{n}\left(X_{1}-e / 2\right)}, & \text { for } X_{1}>e / 2,\end{cases}$ 
with $\left(r_{n}, t_{n}\right)$ the reflection and transmission coefficients of the mode $n$. Now, it is sufficient to apply the jump conditions, Eqs. (14.37), to get $\left(r_{n}, t_{n}\right)$

$$
\left\{\begin{array}{l}
r_{n}=\mathrm{i} \frac{a_{n}-b_{n}}{\left(1-\mathrm{i} a_{n}\right)\left(1-\mathrm{i} b_{n}\right)}, \quad t_{n}=\frac{1+a_{n} b_{n}}{\left(1-\mathrm{i} a_{n}\right)\left(1-\mathrm{i} b_{n}\right)}, \\
\text { with } a_{n} \equiv \frac{k_{n} h}{2}\left[S+C\left(\frac{n \pi}{k_{n} H}\right)^{2}\right], b_{n} \equiv \frac{k_{n} h}{2} B
\end{array}\right.
$$

The interface parameters for this structuration $(e / h=0.05$, $\ell / h=0.95)$ are $: B=2.62[2.64], C=1.510^{-3}\left[\begin{array}{ll}1.7 & \left.10^{-3}\right] \text { and }\end{array}\right.$ $S=2.510^{-3}$.

Figures 14.10 and 14.11 report the wavefields $P^{\mathrm{ex}}(\mathbf{X})$ calculated numerically and $P(\mathbf{X})$ given by the explicit expressions, Eqs. (14.36) to (14.40). The wavefield in the absence of the film for the same incident wave $P^{\text {inc }}\left(X_{1}, X_{2}\right)=P_{n}^{\text {inc }} e^{i k_{n} X_{1}} \varphi_{n}\left(X_{2}\right)$ is reported for comparison (in both cases, $P^{\text {inc }}$ is a combination of the 100 first modes). The fields $P(\mathbf{X})$ and $P^{\mathrm{ex}}(\mathbf{X})$ are found in good agreement (with a relative discrepancy of about $1 \%$ in both cases), which confirms that the jump conditions correctly account for the effect of the structured screen.

Let us now inspect the reflection and transmission coefficients mode by mode. In the numerics, the scattering properties are encapsulated in matrices $\mathrm{T}$ and $\mathrm{R}$ being $N \times N$ matrices. We calculated $\mathrm{R}$ and $\mathrm{T}$ at the interfaces of the film, namely $P_{m}^{\mathrm{ex}}(-e / 2)=$ $\left[\delta_{m n}+\mathrm{R}_{m n}\right] P_{n}^{\text {inc }}(-e / 2)$, and $P_{m}^{\text {ex }}(e / 2)=\mathrm{T}_{m n} P_{n}^{\text {inc }}(-e / 2)$. We have checked that the scattering matrices are essentially diagonal (the relative weight of the off diagonal terms is about $10^{-4}$ ), which confirms that the mode coupling is negligible. Figures 14.12 show the transmission and reflection coefficients $\left(r_{n}, t_{n}\right)$ from Eqs. (14.40) and the diagonal terms $\left(\mathrm{R}_{n n}, \mathrm{~T}_{n n}\right)$ (the scattering properties do not depend on the source, so they are the same in the cases of the Figs. 14.10 and 14.11). The agreement is relatively good, with $1 \%$ and $5 \%$ respectively for the reflection and transmission coefficients, averaged for $n=1, \ldots 100$. 


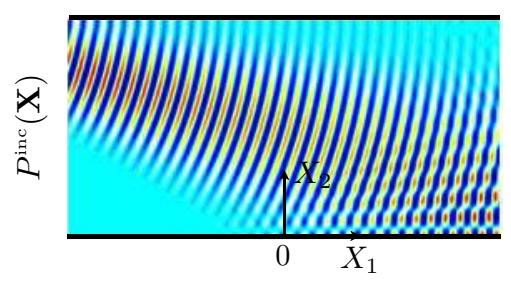

(a)

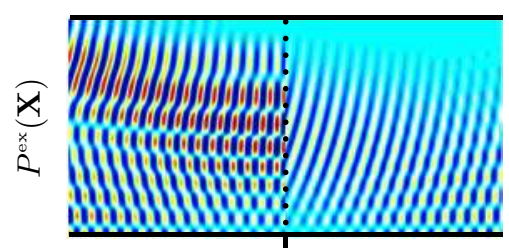

(b)

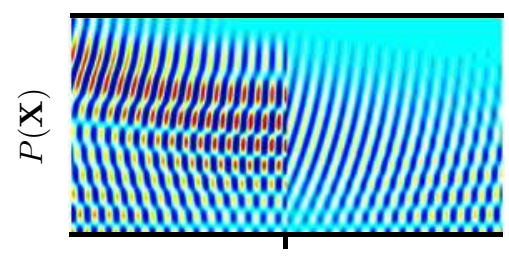

(c)

$\min$

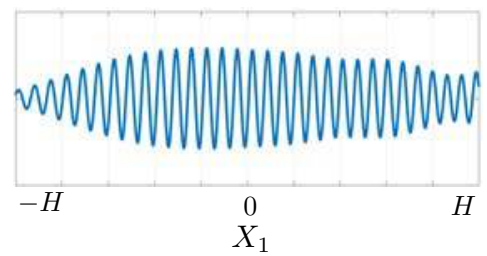

(d)

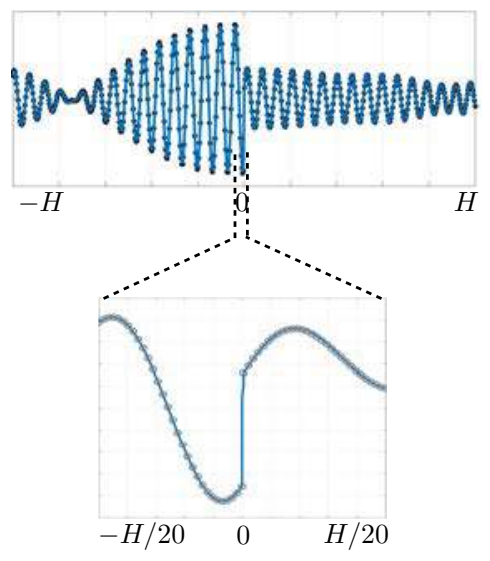

(e)

Fig. 14.10. Scattering by the perforated screen in the guide (geometry of Fig. 14.9). (a) Wavefield $P^{\text {inc }}$ in the absence of the film (b) and (c) Wavefields $P^{\text {ex }}$ calculated numerically and $P$ in the homogenized problem, Eqs. (14.36) to (14.40). (d) shows the profile $P^{\text {inc }}\left(X_{1}, H / 2\right)$ (in arbitrary unit) and (e) show the profiles $P^{\text {ex }}\left(X_{1}, H / 2\right)$ (blue line) and $P\left(X_{1}, H / 2\right)$ (black symbols); the discontinuity of the field across the structured film is visible in the zoom of the profile for $-H / 20<$ $X_{1}<H / 20$.

As can be expected, the full wave calculations have been demanding. Indeed, it requires 3 different scales to be resolved, the largest one $H$, the intermediate one, given by the incident wavenumbers with $k_{n}, n=0$ to 100 , from which the typical wavelength is about $H / 100$, and the smallest one, given by $e=H / 2000$. This latter small scale imposes the truncation $N$ in the numerics, which means that $\left|k_{N}\right| e \sim 1$. Thus $N \pi e / H \sim 1$, leading to $N \sim H / e=2000$. In general, 


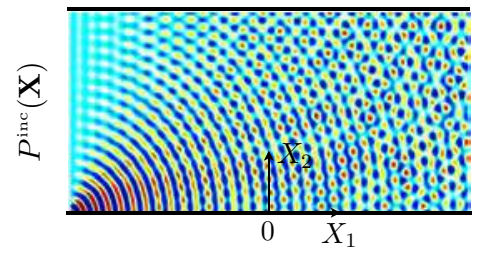

(a)

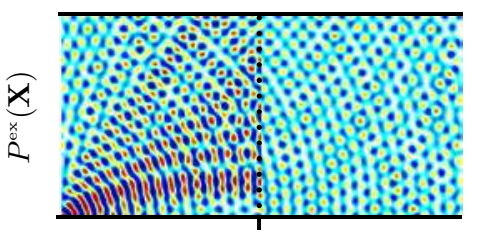

(b)

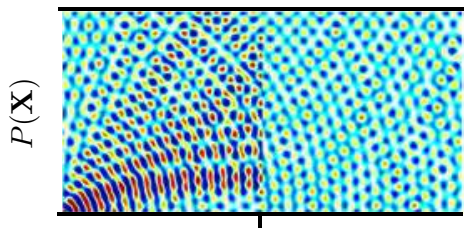

(c)

$\min \max$

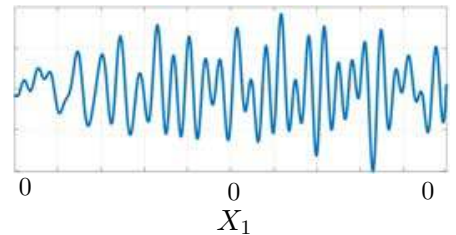

(d)

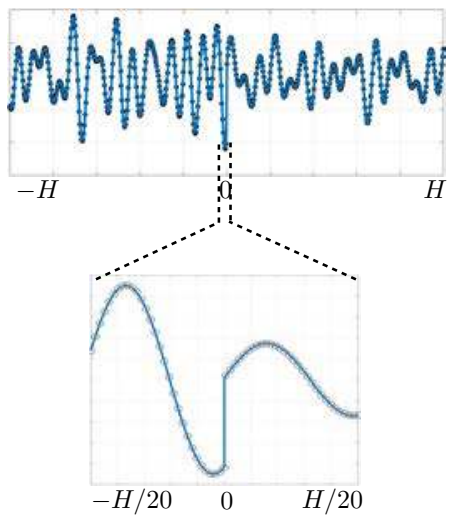

(e)

Fig. 14.11. Same representation as in Fig. 14.10 for an incident wave of beam type.
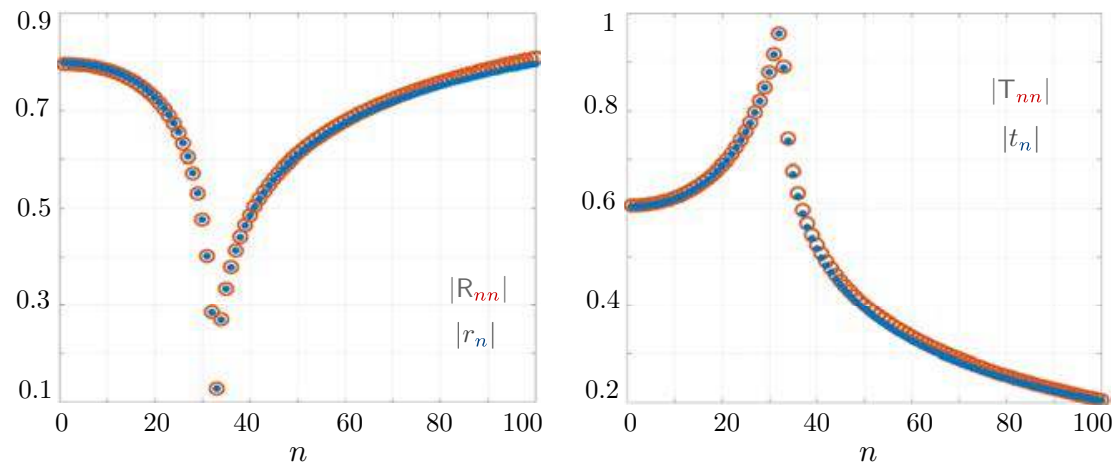

Fig. 14.12. Comparison of the reflection and transmission coefficients of the modes, $\left(\mathrm{R}_{n n}, \mathrm{~T}_{n n}\right)$ for $n=1, \ldots, 100$ from direct numerics (computed with a truncation $N=3000)$, and $\left(r_{n}, t_{n}\right)$ given by the interface model, Eqs. (14.40). 
the smallest scale corresponds to the highest evanescent mode excited in the near field of the structured film, and indeed, $N=2000-3000$ has been needed to reach a reasonably converged solution $P^{\text {ex }}$ (for instance, the variation in $P^{\mathrm{ex}}$, Eq. (14.36), from $N=2000$ to 3000 is about $0.8 \%$ ). This need in a high truncation is expected and not expected. Indeed, it has been said, and it has been checked, that no mode coupling occurs in our configuration (see Appendix 14.9). Thus, in the present cases, the modes at $n>100$ are not expected to contribute significantly to the total field since we have considered an incident wave being a combination of the first 100 modes only. The reason why high truncation is needed is in fact related to the convergence of $\mathrm{R}_{n n}$ and $\mathrm{T}_{n n}$ with $N$, a convergence which is often disregarded; the Appendix 14.9 presents a discussion on this aspect.

\subsection{Concluding Remarks}

We have presented an interface model to identify the effective behavior of a thin structured film composed of sound hard inclusions. Parameters characteristic of the interface enter in jump conditions for the acoustic pressure field and for the acoustic normal velocity. They are given by the resolution of elementary problems written in the static limit, and they are therefore wave independent by construction. We have validated this model in the case of a layer made of rectangular inclusions and for a plane wave at oblique incidence on the structured layer. Explicit expressions of the scattering coefficients deduced from the interface parameters have been shown to be accurate with a range of validity being $k h<1$ and $k e<1$. Results have been compared to the scattering coefficients given by classical homogenization of bulk material; these latter have a range of validity $k h<1$ and $e / h>1$, and largely underestimates the scattering properties of thin structured layers. Finally, the validity and the interest of such jump condition has been exemplified in the more involved case of an ultrathin structured layer obstructing a guide from wall to wall (there, due to the high frequency propagation in the guide, the numerical cost is heavy). 
The present model is applicable to more involved geometries of the inclusions and to inclusions being penetrable for the wave as well as for three dimensional cases. More generally, it can be extended to a large class of wave problems, in acoustics, in elasticity and in electromagnetism.

\subsection{Appendix A - The Present Derivation as a Simplified Case of a Previous One}

In [Marigo and Pideri (2011)], the problem of an elastic body containing microcracks or micro holes periodically located on a surface is considered. The elastic body is submitted to external forces (or boundary conditions) on its boundaries, which impose variations of the external stresses on the scale which is typically the size of the body; this latter is assumed to be much larger than the spacing between the holes. The size of the body in the elastic problem is the equivalent of the incident wavelength in our acoustic problem.

The increased complexity in this work is that it requires to deal with vectors instead of scalars $p, q \rightarrow \mathbf{U}, \mathbf{V}$ (the elastic displacement vector) and a tensor instead of a vector $\mathbf{u}, \mathbf{v} \rightarrow \boldsymbol{\sigma}, \boldsymbol{\tau}$ (the stress tensor). Otherwise, the derivation of the interface parameters is the same.

One noticeable difference is that the calculation of the jump condition are performed after $\tau^{0}$ and $\tau^{1}$ have been extended by 0 in $\mathcal{D}$. This is possible in the static case since the equilibrium translates in $\operatorname{div}_{\mathbf{y}} \boldsymbol{\tau}^{0}=0$ and $\operatorname{div}_{\mathbf{y}} \boldsymbol{\tau}^{1}+\operatorname{div}_{\mathbf{x}^{\prime}} \boldsymbol{\tau}^{0}=0$ with boundary conditions $\boldsymbol{\tau}^{0} \cdot \mathbf{n}_{\mid \partial \mathcal{D}}=\boldsymbol{\tau}^{1} \cdot \mathbf{n}_{\mid \partial \mathcal{D}}=0$. Thus, extending $\boldsymbol{\tau}^{n}$ by 0 in $\mathcal{D}$ is compatible with the equilibrium and makes the boundary conditions on $\partial \mathcal{D}$ to be automatically satisfied. This is not possible in our case, and this is why the integrations are performed in $Y \backslash \mathcal{D}$ instead of in $Y$ in [Marigo and Pideri (2011)]. The two results are of course identical. Namely, the Eq. (40) in this reference reads

$$
\llbracket \sigma_{i 1}^{1} \rrbracket=-\frac{\partial}{\partial x_{\alpha}} \int_{\mathbf{Y}} d \mathbf{y} \tau_{i \alpha}\left(\mathbf{x}^{\prime}, \mathbf{y}\right)
$$


and $\boldsymbol{\tau} \equiv \boldsymbol{\tau}^{0}-\boldsymbol{\sigma}^{0}\left(0, \mathbf{x}^{\prime}\right)$ has been extended implicitly by $-\boldsymbol{\sigma}^{0}\left(0, \mathbf{x}^{\prime}\right)$ in $\mathcal{D}$. It follows that

$$
\llbracket \sigma_{i 1}^{1} \rrbracket=\int_{\mathcal{D}} d \mathbf{y} \frac{\partial \boldsymbol{\sigma}_{i \alpha}^{0}}{\partial x_{\alpha}}\left(0, \mathbf{x}^{\prime}\right)-\frac{\partial}{\partial x_{\alpha}} \int_{\mathbf{Y} \backslash \mathcal{D}} d \mathbf{y} \tau_{i \alpha}\left(\mathbf{x}^{\prime}, \mathbf{y}\right),
$$

and owing to $\frac{\partial \sigma_{i \alpha}^{0}}{\partial x_{\alpha}}=-\frac{\partial \sigma_{i 1}^{0}}{\partial x_{1}}$ given by the outer problem, we get

$$
\llbracket \sigma_{i 1}^{1} \rrbracket=-\frac{\partial \boldsymbol{\sigma}_{i 1}^{0}}{\partial x_{1}}\left(0, \mathbf{x}^{\prime}\right) S-\frac{\partial}{\partial x_{\alpha}} \int_{Y \backslash \mathcal{D}} d \mathbf{y} \tau_{i \alpha}\left(\mathbf{x}^{\prime}, \mathbf{y}\right),
$$

which is equivalent to our jump condition Eq. (14.15). Finally, the elementary problems satisfied in the static elastic case are given by Eqs. (28) are the equivalent of our Eqs. (14.17).

\subsection{Appendix $\mathrm{C}$ - Multimodal Approach to Compute $(B, C)$ for Rectangular Rigid Structuration}

For piecewise constant geometry in the elementary problems, mode matching is a simple way to get $B$ and $C$. In both elementary problems (14.17), the idea is to project the solution $q^{(i)}$ onto bases of transverse functions of $\mathbf{y}^{\prime}$ being adapted in the different regions along $y_{1}$. Below, we give a simple procedure to compute $(B, C)$ for rectangular rigid inclusions in two dimensions (in this case, only two bases of transverse functions $\left(f_{n}^{-}\left(\mathbf{y}^{\prime}\right), f_{n}^{+}\left(\mathbf{y}^{\prime}\right)\right.$ are needed). The simplicity lies in the fact that the procedure does not require more than the inversion of a matrix. The size $N$ of the matrix fixes the spatial resolution : with a cell of size unity along $y_{2}$, the spatial resolution is typically $1 / N$. As we assumed that all the dimensions of the inclusions is of order unity, large $N$ is not required (otherwise, the calculation may become tricky since mode matching has low convergence, typically $1 / N)$.

In the following, we consider $h=1$ for simplicity. 


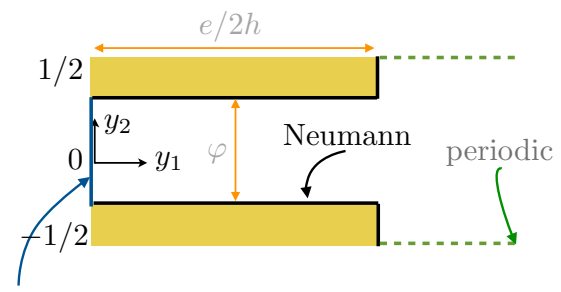

Dirichlet (Pb1), Neumann (Pb2)

Fig. 14.13. Mode matching configuration. The solutions $Q^{-}$and $Q^{+}$are written for $0 \leq y_{1} \leq e / 2 h$ and $y_{1} \geq e / 2 h$ respectively (with proper conditions at $y_{1}=0$ ), and the resolution involves only matching conditions at $y_{1}=e / 2 h$.

\subsubsection{Calculation of $B$ in the elementary problem $i=1$}

Because $q^{(1)}$ is defined up to a constant and because the symmetry of the inclusion w.r.t. $y_{1}$, we can consider a function $q^{(1)}$ odd w.r.t. $y_{1}$, whence applying Dirichlet boundary condition at $y_{1}=0$ (namely $\left.q^{(1)}\left(0, y_{2}\right)=0\right)$. It follows that $q^{(1)} \rightarrow b_{1} / 2$ for $y_{1} \rightarrow+\infty$ (and $\left.b_{1}=\delta q^{(1)}\right)$. We use $Q(\mathbf{y})=y_{1}+q^{(1)}(\mathbf{y})-b_{1} / 2$ which satisfies $\Delta Q=0$, $\boldsymbol{\nabla} Q \cdot \mathbf{n}_{\mid \partial \mathcal{D}}=0$, and $\left(Q-y_{1}\right) \rightarrow 0$ for $y_{1} \rightarrow+\infty$. Finally, we use that $\int_{-\varphi / 2}^{\varphi / 2} \mathrm{~d} y_{2} \partial_{y_{1}} Q\left(0, y_{2}\right)=\int_{-1 / 2}^{1 / 2} \mathrm{~d} y_{2} \partial_{y_{1}} Q\left(+\infty, y_{2}\right)=1$ (by integration of $\Delta Q=0)$. Thus, $Q$ can be expanded as

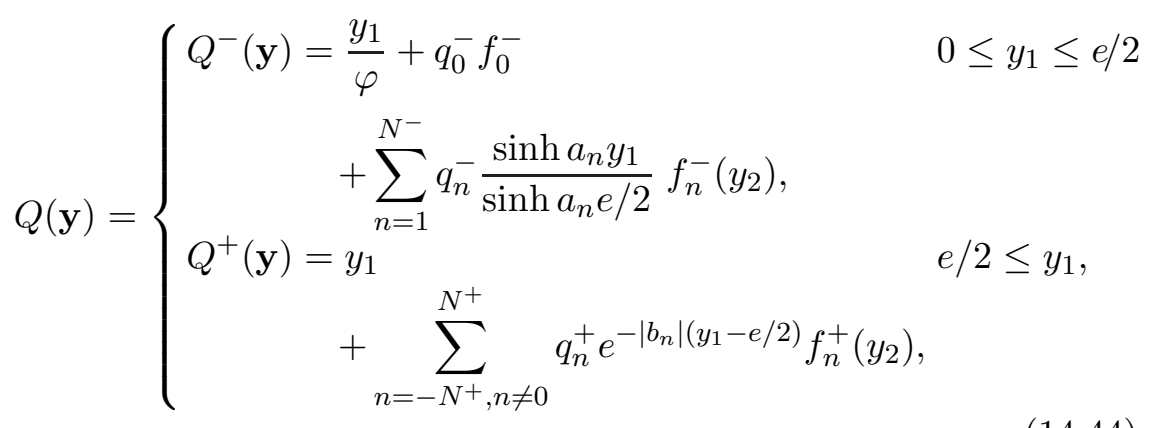

with $a_{n}=n \pi / \varphi, b_{n}=2 n \pi$, and where

$$
f_{n}^{+}\left(y_{2}\right)=e^{\mathrm{i} b_{n} y_{2}}, \quad f_{n}^{-}\left(y_{2}\right)=\sqrt{\frac{2-\delta_{n 0}}{\varphi}} \cos \left(a_{n} y_{2}+\frac{n \pi}{2}\right),
$$


are the transverse functions (forming a basis) adapted for solutions being respectively periodic and for solutions with zero derivatives at $y_{2}= \pm \varphi / 2$.

In the above projection, $b_{1}$ being unknown has ben encapsulated in the first coefficient $q_{0}^{-}$of the expansion, and it is easy to see that $Q^{-}\left(0, y_{2}\right)=q_{0}^{-} f_{0}^{-}=-b_{1} / 2$ (thus $\left.b_{1}=-2 q_{0}^{-} / \sqrt{\varphi}\right)$, by construction.

Now, we will ask to $Q^{ \pm}$to match (on average) their values and their first derivatives at $y_{1}=e / 2$, and this latter matching on the derivatives will be done accounting for the Neumann boundary conditions at $y_{1}=e / 2$ and $\left|y_{2}\right|>\varphi / 2$ (note that $Q^{-}$satisfies by construction the Neumann boundary condition on at $y_{2}= \pm \varphi / 2$, because of the choice of the $f_{m}^{-}$). To that aim, we use the following relations

$$
\left\{\begin{array}{l}
\int_{-\varphi / 2}^{\varphi / 2} \mathrm{~d} y_{2} Q^{-}\left(e / 2, y_{2}\right) f_{m}^{-}\left(y_{2}\right)=\int_{-\varphi / 2}^{\varphi / 2} \mathrm{~d} y_{2} Q^{+}\left(e / 2, y_{2}\right) f_{m}^{-}\left(y_{2}\right), \\
\int_{-\varphi / 2}^{\varphi / 2} \mathrm{~d} y_{2} \frac{\partial Q^{-}}{\partial y_{1}}\left(e / 2, y_{2}\right) f_{m}^{+*}\left(y_{2}\right)=\int_{-1 / 2}^{1 / 2} \mathrm{~d} y_{2} \frac{\partial Q^{+}}{\partial y_{1}}\left(e / 2, y_{2}\right) f_{m}^{+*}\left(y_{2}\right),
\end{array}\right.
$$

with $f_{m}^{+*}$ the conjugate of $f_{m}^{+}\left(f_{m}^{-}\right.$is real $)$. The first relation is the matching of the values in the region $y_{2} \in[-\varphi / 2, \varphi / 2]$ where $Q^{-}$is defined. The second relation has more information: we have used that the $\partial_{y_{1}} Q^{+}=0$ at $y_{1}=e / 2$ for $\left|y_{2}\right|>\varphi / 2$, from which

$$
\int_{-1 / 2}^{1 / 2} \mathrm{~d} y_{2} \frac{\partial Q^{+}}{\partial y_{1}}\left(e / 2, y_{2}\right) f_{n}^{+*}\left(y_{2}\right)=\int_{-\varphi / 2}^{\varphi / 2} \mathrm{~d} y_{2} \frac{\partial Q^{+}}{\partial y_{1}}\left(e / 2, y_{2}\right) f_{m}^{+*}\left(y_{2}\right),
$$

afterwards we ask, on average, $\partial_{y_{1}} Q^{+}=\partial_{y_{1}} Q^{-}$for $\left|y_{2}\right|<\varphi / 2$. We get a matrix system for the two vectors $\mathbf{q}^{-}=\left(q_{n}^{-}\right)_{n=0, \ldots, N^{-}}$and $\mathbf{q}^{+}=\left(q_{n}^{+}\right)_{n=0, \ldots, N^{+}}$of the form

$$
\left(\begin{array}{cc}
\mathrm{I} & -{ }^{t} \mathrm{~F}^{*} \\
\mathrm{FA}_{1} & \mathrm{~B}
\end{array}\right)\left(\begin{array}{l}
\mathbf{q}^{-} \\
\mathbf{q}^{+}
\end{array}\right)=\left(\begin{array}{l}
\mathbf{S}^{s} \\
\mathbf{S}^{d}
\end{array}\right)
$$


with I the $N^{-} \times N^{-}$identity matrix, $\mathrm{A}_{1}=\operatorname{diag}\left(a_{n} \operatorname{cotanh}\left(a_{n} e / 2\right)\right)$ with $\mathrm{A}_{1}(1,1)=0, \mathrm{~B}=\operatorname{diag}\left(\left|b_{n}\right|\right)$, and $F_{m n}=\int_{-\varphi / 2}^{\varphi / 2} \mathrm{~d} y_{2} f_{n}^{+*}\left(y_{2}\right) f_{n}^{-}$ $\left(y_{2}\right)$. The source terms $\left(\mathbf{S}^{s}, \mathbf{S}^{d}\right)$ correspond to the projection of the loading $\left(y_{1} / \varphi\right.$ and $\left.y_{1}\right)$ onto $f_{n}^{-}$and $f_{n}^{+*}$. The expressions of $F_{m n}$ and $S_{n}^{s, d}$ are given below

$$
F_{m n}=\left\{\begin{array}{rlrl}
\sqrt{\varphi} \operatorname{sinc}\left(b_{m} \frac{\varphi}{2}\right), & n=0 \\
= & \sqrt{\frac{\varphi}{2}}\left[\operatorname{sinc}\left(\left(a_{n}-b_{m}\right) \frac{\varphi}{2}\right) e^{\mathrm{i} n \pi / 2}\right. & & n \neq 0, \\
& \left.+\operatorname{sinc}\left(\left(a_{n}+b_{m}\right) \frac{\varphi}{2}\right) e^{-\mathrm{i} n \pi / 2}\right], &
\end{array}\right.
$$

and

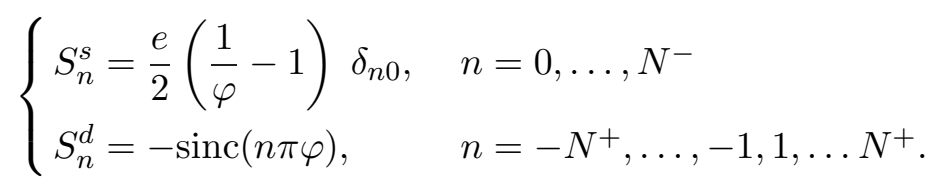

The system is of the form $\mathbf{M q}=\mathbf{s}$ with the matrix $M$ being square (this is not always the case in systems written using mode matching). Next, $\mathrm{M}$ is invertible if one has taken care to consider only the antisymmetric modes. Thus, the system can be solved to find $\mathbf{q}$ by inverting $\mathrm{M}$ or it can be solved in the least squares sense (as done by the operation $\mathbf{M} \backslash \mathbf{s}$ in Matlab). Once the system has been inverted, $b_{1}$ is obtained and thus, from (14.28), $B=e+b_{1}$

$$
B=e-\frac{2}{\varphi} q(1) .
$$

\subsubsection{Calculation of $C$ in the elementary problem $i=2$}

The procedure to get $C$ in the elementary problem $i=2$ is similar. We consider the solution $Q=Q^{(2)}+y_{2}$ satisfying $\Delta Q=0$, 
$\boldsymbol{\nabla} Q . \mathbf{n}_{\mid \partial \mathcal{D}}=0$ and $Q \rightarrow y_{2}$ for $y_{1} \rightarrow \infty$. Because $q^{(2)}$ is even in $y_{1}$, we can project $Q$ onto the bases $\left(f_{n}^{ \pm}\right)$as follow

$$
Q(\mathbf{y})=\left\{\begin{array}{rlr}
Q^{-}(\mathbf{y})=\sum_{n=1}^{N^{-}} q_{n}^{-} \frac{\cosh a_{n} y_{1}}{\cosh a_{n} e / 2} f_{n}^{-}\left(y_{2}\right), & & -e / 2 \leq y_{1} \leq 0 \\
Q^{+}(\mathbf{y})= & y_{2} & \\
& +\sum_{n=-N^{+}, n \neq 0}^{N^{+}} q_{n}^{+} e^{-\left|b_{n}\right| y_{1}} f_{n}^{+}\left(y_{2}\right), & 0 \leq y_{1} .
\end{array}\right.
$$

As previously, we ask to $Q^{ \pm}$to match (on average) their values and their first derivatives at $y_{1}=e / 2$, and we get a matrix system of the form

$$
\left(\begin{array}{cc}
\mathrm{I} & -{ }^{t} \mathrm{~F}^{*} \\
\mathrm{FA}_{2} & \mathrm{~B}
\end{array}\right)\left(\begin{array}{l}
\mathbf{q}^{-} \\
\mathbf{q}^{+}
\end{array}\right)=\left(\begin{array}{l}
\mathbf{S} \\
\mathbf{0}
\end{array}\right)
$$

with the same definitions as previously and now, $\mathrm{A}_{2}=$ $\operatorname{diag}\left(a_{n} \tanh \left(a_{n} e / 2\right)\right)$ and $S_{n}=\int_{-\varphi / 2}^{\varphi / 2} \mathrm{~d} y_{2} y_{2} f_{n}^{-}\left(y_{2}\right)$, specifically

$$
S_{n}=-2 \sqrt{\frac{2}{\varphi}} \frac{1}{a_{n}^{2}} .
$$

Once the system has been inverted, we can determine

$$
C=-\int \mathrm{d} \mathbf{y} \frac{\partial Q^{(2)}}{\partial y_{2}}=\int_{-e}^{0} \mathrm{~d} y_{1} \int_{-\varphi / 2}^{\varphi / 2}\left[1-\frac{\partial Q^{-}}{\partial y_{2}}\right] .
$$

where we have used that $Q^{(2)}\left(y_{1} \geq 0, y_{2}\right)=Q^{+}(\mathbf{y})-y_{2}$ is periodic, thus of vanishing contribution. It is now sufficient to write $C=e \varphi-$ $q_{n}^{-} \tanh a_{n} e / a_{n}\left[f_{n}\right]_{-\varphi / 2}^{\varphi / 2}$ to get

$$
C=e \varphi+2 \sqrt{\frac{2}{\varphi}} \frac{\tanh a_{n} e}{a_{n}} q_{n}^{-} .
$$




\subsubsection{Scripts in Matlab}

Simple scripts providing $(B, C)$ are given below.

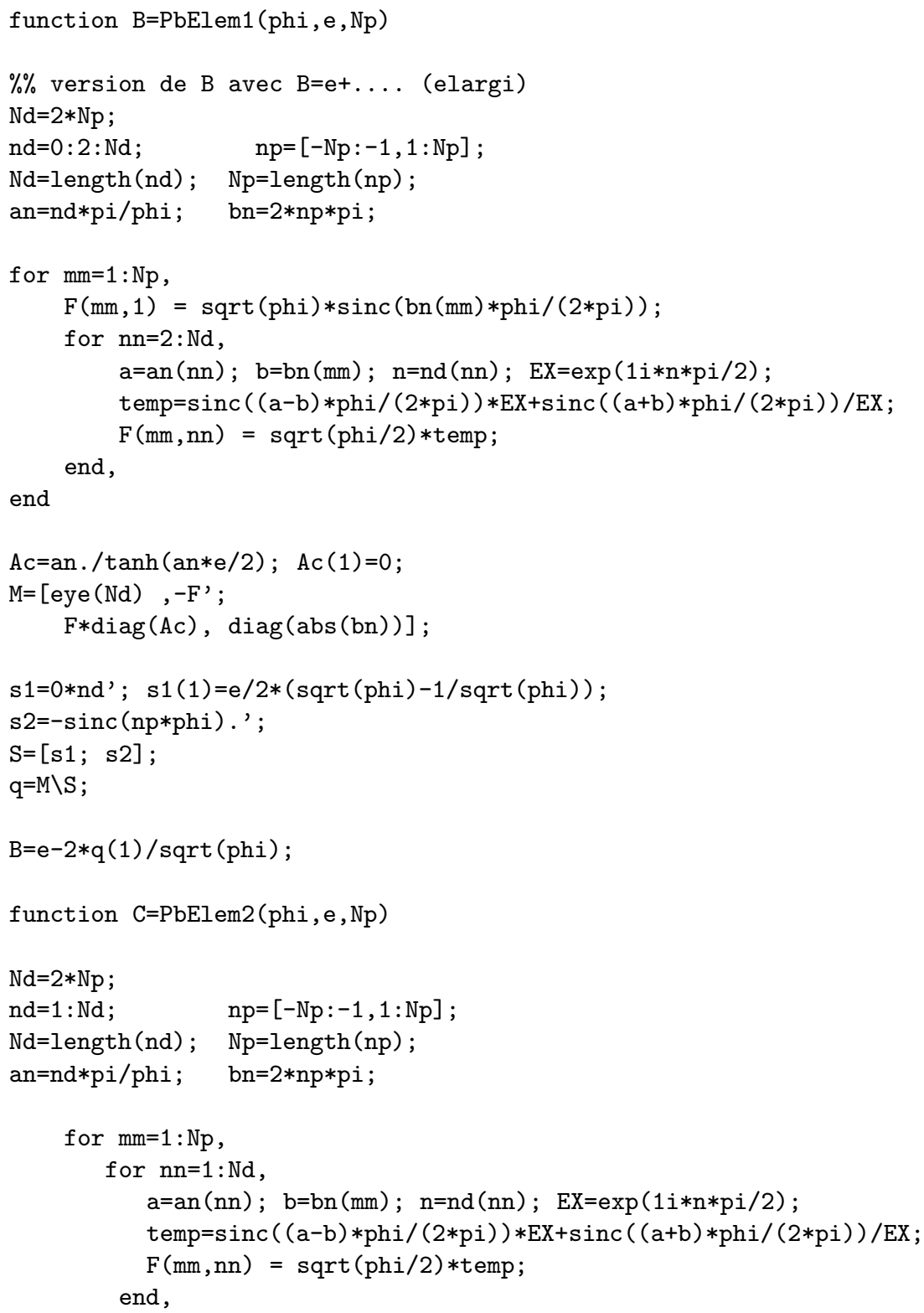




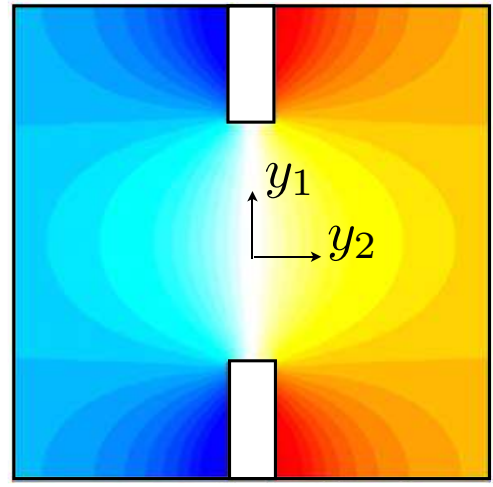

(a)

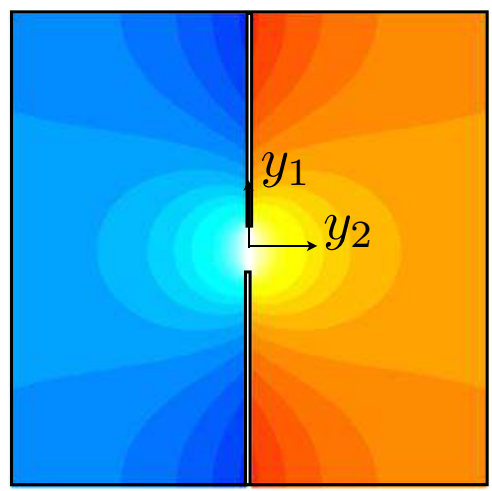

(c)

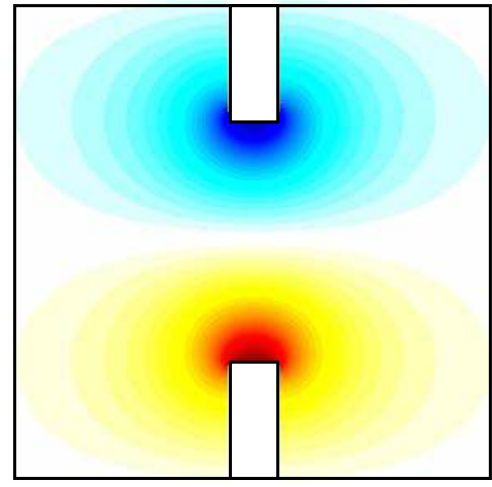

(b)



(d)

Fig. 14.14. Static fields computed for the elementary problems $i=1$ and 2 . (a) (c) $q^{(1)}(\mathbf{y})$ and (b) (d) $q^{(2)}(\mathbf{y})$. (a-b) for $e / h=0.1$ and $\ell / h=0.5$, (c-d) for $e / h=0.01$ and $\ell / h=0.9$.

end

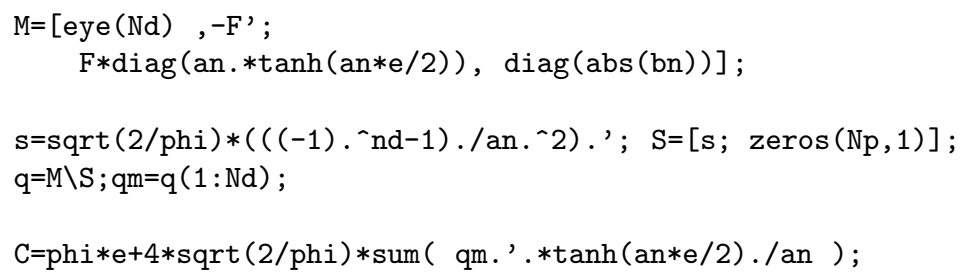




\subsection{Appendix D - Reflection Coefficient Using the Classical Homogenization}

In this Appendix, we consider $h=1$ for simplicity.

\subsubsection{Scattering coefficients in the classical homogenization}

Classical homogenization relies on the same expansion of the field in power of $\varepsilon$. Let us consider penetrable inclusions presenting a contrast in mass density and a contrast in bulk modulus with respect to the surrounding medium. In a general way, the wave propagation is the equation of propagation in inhomogeneous media

$$
\operatorname{div}[a(\mathbf{X}) \boldsymbol{\nabla} P]+b(\mathbf{X}) P=0,
$$

with $a$ the inverse of the mass density and $b$ the inverse of the bulk modulus; we denote $a(\mathbf{X})=a, b(\mathbf{X})=b$ inside the inclusion and $a(\mathbf{X})=b(\mathbf{X})=1$ in the surrounding medium. At the inclusion boundaries $\partial \mathcal{D}, P$ and $a(\mathbf{X}) \partial_{n} P$ are continuous. For acoustic waves, It is easy to see that applying $a=b=0$ in the inclusions leads to the Neumann limit, with $\Delta P+P=0$ in the surrounding medium and $\partial_{n} P_{\mid \partial \mathcal{D}}=0$. This is consistent with the intuitive idea that Neumann boundary condition corresponds to heavy materials, with infinite mass density and infinite bulk modulus. At leading order, $P \simeq P_{0}$ satisfies

$$
\operatorname{div}\left[\mathrm{a}_{\mathrm{eff}} \boldsymbol{\nabla} P\right]+b_{\mathrm{eff}} P=0,
$$

with the tensor $a_{\text {eff }}$ and $b_{\text {eff }}$ given by the resolution of two elementary problems. The case of layered media, with inclusions infinitely long along $X_{1}$ and of width $\ell$ along $X_{2}$, we have $a\left(X_{2}\right)$ and $b\left(X_{2}\right)$ and explicit solution is possible. It ends with diagonal effective mass density tensor $a_{\text {eff }}=\operatorname{diag}\left(a_{\text {eff }}^{1}, a_{\text {eff }}^{2}\right)$ and effective bulk modulus given by

$$
\left\{\begin{array}{l}
a_{\mathrm{eff}}^{1}=\left\langle a\left(X_{2}\right)\right\rangle=[\ell a+(1-\ell)] \rightarrow 1-\ell, \\
a_{\mathrm{eff}}^{2}=\left\langle a\left(X_{2}\right)^{-1}\right\rangle^{-1}=[\ell / a+(1-\ell)]^{-1} \rightarrow 0, \\
b_{\mathrm{eff}}=\left\langle b\left(X_{2}\right)\right\rangle=[\ell b+(1-\ell)] \rightarrow 1-\ell,
\end{array}\right.
$$


where the limits correspond to the Neumann case $(a=b=0)$. In this limit, the propagation inside the layered medium is described by the wave equation $a_{\mathrm{eff}}^{1} \partial_{X_{1}^{2}} P+a_{\mathrm{eff}}^{2} \partial_{X_{2}^{2}} P+k^{2} b_{\mathrm{eff}} P=(1-\ell)\left[\partial_{X_{1}^{2}} P+k^{2} P\right]=$ 0 , from which we deduce that the component of the wavenumber along $X_{1}$ is $k$.

Imagine now that we cut the layered medium to create a slab, which occupies the space $X_{1} \in[-e / 2, e / 2]$, and outside the slab, the medium has $a(\mathbf{X})=b(\mathbf{X})=1$. For the real structure, the resulting configuration corresponds to an array of rectangular hard inclusions as studied in Section 14.2. For the equivalent homogenized medium, it corresponds to a slab of thickness $e$ filled with the homogeneous and anisotropic material given by $\left(a_{\text {eff }}, b_{\text {eff }}\right)$. In this latter configuration, the problem of the scattering for an incident wave coming from $X_{1}=$ $-\infty$ with incidence $\theta$ is trivial and it has a solution of the form

$$
\begin{aligned}
& P\left(X_{1}, X_{2}\right) \\
& =e^{\mathrm{i} k \sin \theta X_{2}} \begin{cases}{\left[e^{\mathrm{i} k \cos \theta X_{1}}+\tilde{R} e^{-\mathrm{i} k \cos \theta\left(X_{1}+e / 2\right)}\right],} & \text { for } X_{1}<-e / 2, \\
{\left[A e^{\mathrm{i} k X_{1}}+B e^{-\mathrm{i} k X_{1}}\right],} & \text { for }\left|X_{1}\right| \leq e / 2, \\
\tilde{T} e^{\mathrm{i} k \cos \theta\left(X_{1}-e / 2\right)}, & \text { for } X_{1}>e / 2,\end{cases}
\end{aligned}
$$

where the component of the wavenumber along $X_{2}, k \sin \theta$, is imposed by the continuity of $P\left(X_{1}, X_{2}\right)$ at the interfaces $X_{1}= \pm e / 2$. Next, applying the relations of continuity for $P$ and $a_{1}(\mathbf{X}) \partial_{X_{1}} P$ (with $a_{1}(\mathbf{X})=a_{\mathrm{eff}}^{1}=1-\ell$ in the slab and $a_{1}(\mathbf{X})=1$ outside) at $X_{1}= \pm e / 2$, leads to usual forms of the scattering coefficients

$$
\tilde{R}=\frac{2 \mathrm{i}\left(1-c^{2}\right) \sin k e e^{-\mathrm{i} k e \cos \theta / 2}}{(1+c)^{2} e^{-\mathrm{i} k e}-(1-c)^{2} e^{\mathrm{i} k e}}, \quad \tilde{T}=\frac{4 c e^{-\mathrm{i} k e \cos \theta / 2}}{(1+c)^{2} e^{-\mathrm{i} k e}-(1-c)^{2} e^{\mathrm{i} k e}},
$$

where $c \equiv \cos \theta /(1-\ell)$. The form of $\tilde{R}$ in the above equation is written in an alternative form in Eq. (14.34). 


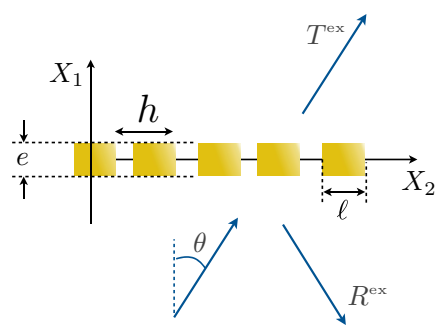

(a)

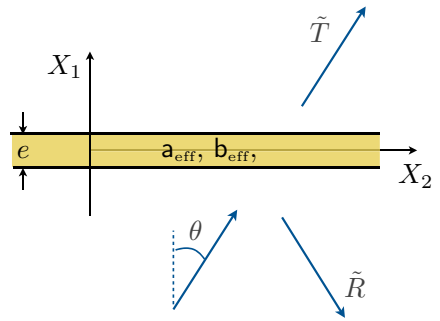

(b)

Fig. 14.15. In the classical homogenization, the actual structured film (a) is replaced by a homogeneous anisotropic film of thickness $e$.

The derivation of $(\tilde{R}, \tilde{T})$ is done accounting for boundary conditions being the continuity of $p$ and of $\mathrm{a}_{\mathrm{eff}} \boldsymbol{\nabla} p \cdot \mathbf{n}$, which is true at this leading order (see [Marigo and Maurel (2017)]).

\subsubsection{Jump conditions in the classical homogenization}

It is tempting to inspect if classical homogenization gives jump conditions similar to those obtained from our interface model, Eq.(14.29). To that end, we consider, from Eqs. (14.60) the quantities $\llbracket P \rrbracket=$ $\tilde{T}-\tilde{R}-1, \llbracket \partial_{X_{1}} P \rrbracket=i k \cos \theta(\tilde{T}+\tilde{R}-1)$, with $\overline{\partial_{X_{1}} P}\left(0, X_{2}\right)=$ $i k \cos \theta(\tilde{T}-\tilde{R}+1) / 2, \overline{\partial_{X_{1}^{2}} P}\left(0, X_{2}\right)=(i k \cos \theta)^{2}(\tilde{T}-\tilde{R}+1) / 2$ and $\overline{\partial_{X_{2}^{2}} P}\left(0, X_{2}\right)=(i k \sin \theta)^{2}(\tilde{T}+\tilde{R}+1) / 2$. Expanding at leading order $(\tilde{T}, \tilde{R})$ in Eqs. (14.61) for small $k e$, it is easy to see that we get

$$
\begin{gathered}
\llbracket P \rrbracket=\frac{e \ell}{1-\ell} \frac{\overline{\partial P}}{\partial X_{1}}\left(0, X_{2}\right), \\
\llbracket \frac{\partial P}{\partial X_{1}} \rrbracket=-e \ell \frac{\partial^{2} P}{\partial X_{1}^{2}}\left(0, X_{2}\right)+e(1-\ell) \frac{\overline{\partial^{2} P}}{\partial X_{2}^{2}}\left(0, X_{2}\right) .
\end{gathered}
$$

This can be written in the form of interface parameters

$$
\tilde{B}=\frac{e \ell}{1-\ell}, \quad \tilde{C}=e(1-\ell), \quad \tilde{S}=e \ell .
$$

While $S=\tilde{S}$ (in the non enlarged version of the jump conditions), both $\tilde{C}$ and $\tilde{B}$ differ from $C, B$ in Eqs. (14.30)-(14.31). The most 
evident difference is that the interface parameters given by classical homogenization misses the blockage coefficients $B_{0}$ which precisely becomes dominant for $e \rightarrow 0$. Next, $C$ and $\tilde{C}$ differ from the correction $-\pi / 8(1-\ell)^{2}$ appearing for large enough thickness. Both corrections are due to boundary film effect, see [Marigo and Maurel (2016)].

\subsection{Appendix $\mathrm{E}$ - Scattering Coefficients for the Modes in a Waveguide}

If the film of the equivalent anisotropic medium occupies the space $X_{1} \in[-e / 2, e / 2]$ and $X_{2} \in[0, H]$ as considered in the Section 14.3.3, it is possible to calculate the reflection and transmission coefficients $\left(\tilde{r}_{n}, \tilde{t}_{n}\right)$ mode by mode (and a mode is defined in Eq. (14.36)). This is because no mode coupling occurs in this case, again owing to the orthogonality of the transverse functions. The problem first reads

$$
a_{\mathrm{eff}}^{1} \frac{\partial^{2} P}{\partial X_{1}^{2}}+a_{\mathrm{eff}}^{2} \frac{\partial^{2} P}{\partial X_{2}^{2}}+k^{2} b_{\mathrm{eff}} p=0
$$

in the film and $\Delta P+k^{2} P=0$ outside, and the relations of continuity of $P$ and $a_{1} \partial_{X_{1}} P$ apply at $X_{1}= \pm e / 2$ (with $a_{1}=a_{\text {eff }}^{1}$ inside the layer, $a_{1}=1$ outside). Expanding the solution into the basis of transverse functions, $P\left(X_{1}, X_{2}\right)=\sum_{n \geq 0} P_{n}\left(X_{1}\right) \varphi_{n}\left(X_{2}\right)$, the system is decoupled, with

$$
\begin{cases}a_{\mathrm{eff}}^{1} P_{n}^{\prime \prime}+\left[k^{2} b_{\mathrm{eff}}-a_{\mathrm{eff}}^{2}\left(\frac{n \pi}{H}\right)^{2}\right] P_{n}=0, & \text { for }\left|X_{1}\right| \leq e / 2 \\ P_{n}^{\prime \prime}+\left[k^{2}-\left(\frac{n \pi}{H}\right)^{2}\right], & \text { outside }\end{cases}
$$

with $P_{n}$ continuous at $X_{1}= \pm e / 2$, and $P_{n}^{\prime}\left(-e / 2^{-}\right)=a_{\mathrm{eff}}^{1} P_{n}^{\prime}\left(-e / 2^{+}\right)$, $P_{n}^{\prime}\left(e / 2^{+}\right)=a_{\text {eff }}^{1} P_{n}^{\prime}\left(e / 2^{-}\right)$. In the case of our layered sound hard medium, $a_{\text {eff }}^{1}=b_{\text {eff }}=1-\ell$ and $a_{\text {eff }}^{2}=0$, which leads to $P_{n}^{\prime \prime}+k^{2} P_{n}=0$ in the layer, $\left|X_{1}\right| \leq e / 2$. Thus, the problem is identical to the previous 
one (Eqs. (14.60)), owing to $k \cos \theta \rightarrow k_{n}$ and

$$
\begin{aligned}
& \tilde{r}_{n}=\frac{2 \mathrm{i}\left(1-c_{n}^{2}\right) \sin k e e^{-\mathrm{i} k_{n} e / 2}}{\left(1+c_{n}\right)^{2} e^{-\mathrm{i} k e}-\left(1-c_{n}\right)^{2} e^{\mathrm{i} k e}}, \\
& \tilde{t}_{n}=\frac{4 c_{n} e^{-\mathrm{i} k_{n} e / 2}}{\left(1+c_{n}\right)^{2} e^{-\mathrm{i} k e}-\left(1-c_{n}\right)^{2} e^{\mathrm{i} k e}},
\end{aligned}
$$

with $c_{n} \equiv k_{n} / k(1-\ell)$.

\subsection{Appendix F - Discussion on the Convergence of the Scattering Coefficients in the Modal Method}

In the problem considered in Section 14.3.3, we have said that the high truncation $N=2000-3000$ was expected and not expected. Expected because the smallest scale to be resolved is $e=H / 2000$ which imposes $N \sim H / e$ (and this corresponds to the highest evanescent mode with wavenumber $\left.k_{N} \sim N \pi / H\right)$. Unexpected because we have checked that the mode coupling is negligible; the incident wave being a combination of the first 100 modes, the absence of mode coupling ensures that the modes at $n>100$ are not excited. To be clear, we have compared $P^{\mathrm{ex}}(\mathbf{X})$ computed with 3000 modes to the following field

$$
P^{\operatorname{diag}}(\mathbf{X})= \begin{cases}\sum_{n=0}^{100} P_{n}^{\mathrm{inc}}\left[e^{\mathrm{i} k_{n}\left(X_{1}+e / 2\right)}+\mathrm{R}_{n n} e^{-\mathrm{i} k_{n}\left(X_{1}+e / 2\right)}\right], & X_{1}<-e / 2, \\ \sum_{n=0}^{100} P_{n}^{\mathrm{inc}} \mathrm{T}_{n n} e^{\mathrm{i} k_{n}\left(X_{1}-e / 2\right)}, & X_{1}>e / 2,\end{cases}
$$

which is precisely the field composed of the 100 first modes generated directly by the incident wave. The difference $\left|P^{\text {ex }}-P^{\text {diag }}\right|$ is less than $0.4 \%$ in both cases, thus the evanescent field generated by higher modes is indeed negligible. The reason why high truncation 


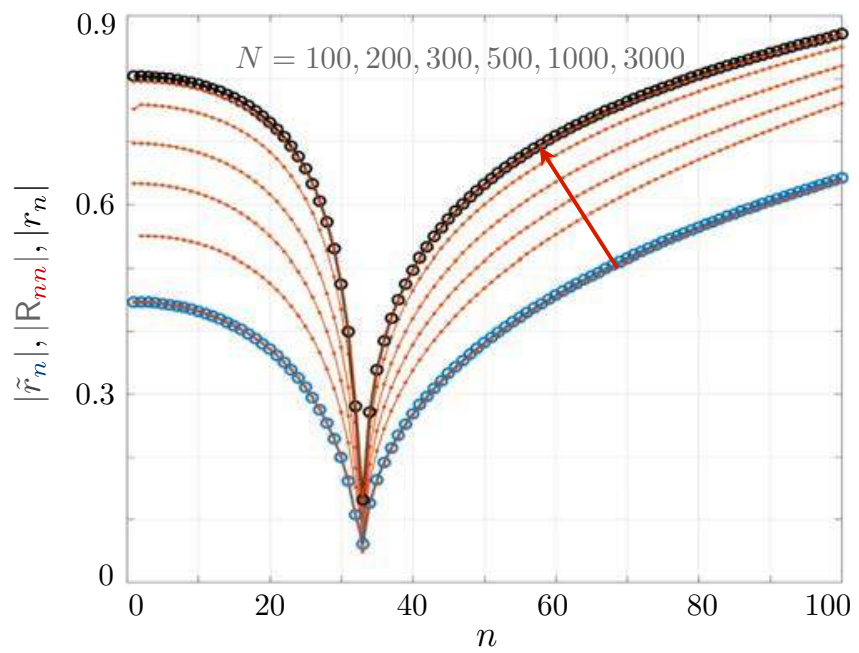

Fig. 14.16. Reflection coefficients of the modes in the guide obstructed by a perforated screen (same calculations as in Fig. 14.12). $\left|\mathrm{R}_{n n}\right|$ for $n=0$ to 100 is shown for several truncation order $N$ in the expansion Eq. (14.36) (orange curves). For low truncation, $N=100,\left|\mathrm{R}_{n n}\right|$ are found to be close to the $\tilde{r}_{n}$ given by the classical homogenization in (14.66) (blue symbols); increasing $N$ makes $\left|\mathrm{R}_{n n}\right|$ to converge toward $r_{n}$ given by the interface homogenization, $\left|r_{n}\right|$ in Eq. (14.40) (black symbols).

has been needed is in fact related to the convergence of $\mathrm{R}_{n n}$ and $\mathrm{T}_{n n}$ for $n \leq 100$ with $N$, a convergence which is often disregarded. In Fig. 14.16, we have reported the variation of $\left|\mathrm{R}_{n n}\right|, n \leq 100$, for several truncations $N=100$ to 3000 . As it can be seen, increasing $N$ produces a significant variation in $\left|\mathrm{R}_{n n}\right|, 70 \%$ on average for increasing the truncation $N$ from $N=100$ to $N=3000$. This low convergence has already been observed for inclusions presenting a contrast in the mass density [Maurel et al. (2014)]; it is a convergence law in $1 / N$, due to the low regularity of the field (with discontinuous pressure gradient). Amusingly, for insufficient truncation $N=100$, the scattering coefficients coincide with the solution $\left(\tilde{r}_{n}, \tilde{t}_{n}\right)$ given by classical homogenization, which underestimates the weight of the evanescent field (Eqs. (14.66), see Appendix 14.8). 


\section{Bibliography}

[1] Abdelmoula, R., Coutris, M. and Marigo, J.-J. (1998). Comportement asymptotique d'une interphase élastique mince, Comptes Rendus de l'Académie des Sciences-Series IIB-Mechanics-Physics-Chemistry-Astronomy 326, 4, pp. 237-242.

[2] Akarid, A., Ourir, A., Maurel, A., Félix, S. and Mercier, J.-F. (2014). Extraordinary transmission through subwavelength dielectric gratings in the microwave range, Optics Letters 39, 13, pp. 3752-3755.

[3] Antonakakis, T., Craster, R. and Guenneau, S. (2013). Asymptotics for metamaterials and photonic crystals, in Proceedings of the Royal Society of London A: Mathematical, Physical and Engineering Sciences, Vol. 469 (The Royal Society), p. 20120533.

[4] Arslanagic, S., Hansen, T. V., Mortensen, N. A., Gregersen, A. H., Sigmund, O., Ziolkowski, R. W. and Breinbjerg, O. (2013). A review of the scatteringparameter extraction method with clarification of ambiguity issues in relation to metamaterial homogenization, Antennas and Propagation Magazine, IEEE 55, 2, pp. 91-106.

[5] Bonnet-Bendhia, A., Drissi, D. and Gmati, N. (2004). Simulation of muffler's transmission losses by a homogenized finite element method, Journal of Computational Acoustics 12, 03, pp. 447-474.

[6] Bouchitté, G. and Felbacq, D. (2004). Homogenization near resonances and artificial magnetism from dielectrics, Comptes Rendus Mathematique 339, 5, pp. 377-382.

[7] Capdeville, Y., Guillot, L. and Marigo, J.-J. (2010a). 1-d non-periodic homogenization for the seismic wave equation, Geophysical Journal International 181, 2, pp. 897-910.

[8] Capdeville, Y., Guillot, L. and Marigo, J.-J. (2010b). 2-d non-periodic homogenization to upscale elastic media for p-sv waves, Geophysical Journal International 182, 2, pp. 903-922.

[9] Capdeville, Y. and Marigo, J.-J. (2007). Second order homogenization of the elastic wave equation for non-periodic layered media, Geophysical Journal International 170, 2, pp. 823-838.

[10] Capdeville, Y. and Marigo, J.-J. (2013). A non-periodic two scale asymptotic method to take account of rough topographies for 2-d elastic wave propagation, Geophysical Journal International 192, 1, pp. 163-189.

[11] Castanié, A., Mercier, J.-F., Felix, S. and Maurel, A. (2014). Generalized method for retrieving effective parameters of anisotropic metamaterials, Optics Express 22, 24, pp. 29937-29953.

[12] Craster, R. V., Kaplunov, J., Nolde, E. and Guenneau, S. (2011). Highfrequency homogenization for checkerboard structures: defect modes, ultrarefraction, and all-angle negative refraction, JOSA A 28, 6, pp. 1032-1040.

[13] David, M., Marigo, J.-J. and Pideri, C. (2012). Homogenized interface model describing inhomogeneities located on a surface, Journal of Elasticity 109, 2, pp. 153-187. 
[14] Delourme, B. (2010). Modèles et asymptotiques des interfaces fines et périodiques en électromagnétisme, Ph.D. thesis, Université Pierre et Marie Curie-Paris VI.

[15] Delourme, B., Haddar, H. and Joly, P. (2012). Approximate models for wave propagation across thin periodic interfaces, Journal de Mathématiques Pures et Appliquées 98, 1, pp. 28-71.

[16] Dimitriadis, A., Sounas, D. L., Kantartzis, N. V., Caloz, C., Tsiboukis, T. D., et al. (2012). Surface susceptibility bianisotropic matrix model for periodic metasurfaces of uniaxially mono-anisotropic scatterers under oblique te-wave incidence, Antennas and Propagation, IEEE Transactions on 60, 12, pp. 5753-5767.

[17] Felbacq, D. and Bouchitté, G. (2005). Left-handed media and homogenization of photonic crystals, Optics Letters 30, 10, pp. 1189-1191.

[18] Flagg, C. and Newman, J. (1971). Sway added-mass coefficients for rectangular profiles in shallow water, Journal of Ship Research 15, p. 4.

[19] Garcia-Vidal, F., Martin-Moreno, L. and Pendry, J. (2005). Surfaces with holes in them: New plasmonic metamaterials, Journal of Optics A: Pure and Applied Optics 7, 2, p. S97.

[20] Guillot, L., Capdeville, Y. and Marigo, J.-J. (2010). 2-d non-periodic homogenization of the elastic wave equation: Sh case, Geophysical Journal International 182, 3, pp. 1438-1454.

[21] Holloway, C. L., Dienstfrey, A., Kuester, E. F., O'Hara, J. F., Azad, A. K. and Taylor, A. J. (2009). A discussion on the interpretation and characterization of metafilms/metasurfaces: The two-dimensional equivalent of metamaterials, Metamaterials 3, 2, pp. 100-112.

[22] Holloway, C. L., Mohamed, M., Kuester, E. F., Dienstfrey, A., et al. (2005). Reflection and transmission properties of a metafilm: With an application to a controllable surface composed of resonant particles, Electromagnetic Compatibility, IEEE Transactions on 47, 4, pp. 853-865.

[23] Kakuno, S., Oda, K. and Liu, P. L. (1992). Scattering of water waves by vertical cylinders with a backwall, Coastal Engineering Proceedings 1, p. 23.

[24] Kelders, L., Allard, J. F. and Lauriks, W. (1998). Ultrasonic surface waves above rectangular-groove gratings, The Journal of the Acoustical Society of America 103, 5, pp. 2730-2733.

[25] Kuester, E. F., Mohamed, M., Piket-May, M., Holloway, C. L., et al. (2003). Averaged transition conditions for electromagnetic fields at a metafilm, Antennas and Propagation, IEEE Transactions on 51, 10, pp. 2641-2651.

[26] Lemoult, F., Kaina, N., Fink, M. and Lerosey, G. (2013). Wave propagation control at the deep subwavelength scale in metamaterials, Nature Physics $\mathbf{9}$, 1 , pp. 55-60.

[27] Li, Y., Jiang, X., Li, R.-q., Liang, B., Zou, X.-y., Yin, L.-l. and Cheng, J.-c. (2014). Experimental realization of full control of reflected waves with subwavelength acoustic metasurfaces, Physical Review Applied 2, 6, p. 064002.

[28] Ma, G., Yang, M., Xiao, S., Yang, Z. and Sheng, P. (2014). Acoustic metasurface with hybrid resonances, Nature Materials. 
[29] Marigo, J. J. and Maurel, A. (2017). Second order Homogenization of subwavelength stratified media including finite size effect, SIAM Journal on Applied Mathematics 77(2), pp. 721-743.

[30] Marigo, J. J. and Maurel, A. (2016). Homogenization models for thin rigid structured surfaces and films, The Journal of the Acoustical Society of America 140(1), pp. 260-273.

[31] Marigo, J.-J. and Pideri, C. (2011). The effective behavior of elastic bodies containing microcracks or microholes localized on a surface, International Journal of Damage Mechanics, p. 1056789511406914.

[32] Martin, P. A. and Dalrymple, R. A. (1988). Scattering of long waves by cylindrical obstacles and gratings using matched asymptotic expansions, Journal of Fluid Mechanics 188, pp. 465-490.

[33] Maurel, A., Félix, S. and Mercier, J.-F. (2013). Enhanced transmission through gratings: Structural and geometrical effects, Physical Review B 88, 11 , p. 115416.

[34] Maurel, A. and Mercier, J.-F. (2012). Propagation of guided waves through weak penetrable scatterers, The Journal of the Acoustical Society of America 131, 3, pp. 1874-1889.

[35] Maurel, A., Mercier, J.-F. and Félix, S. (2014). Wave propagation through penetrable scatterers in a waveguide and through a penetrable grating, The Journal of the Acoustical Society of America 135, 1, pp. 165-174.

[36] Mercier, J., Cordero, M., Félix, S., Ourir, A. and Maurel, A. (2015). Classical homogenization to analyze the dispersion relations of spoof plasmons with geometrical and compositional effects, Applied Physics Letters, submitted.

[37] Morse, P. M. and Ingard, K. U. (1968). Theoretical Acoustics (Princeton University Press).

[38] Oleinik, O. A., Shamaev, A. and Yosifian, G. (2009). Mathematical Problems in Elasticity and Homogenization, Vol. 2 (Elsevier).

[39] Pendry, J., Martin-Moreno, L. and Garcia-Vidal, F. (2004). Mimicking surface plasmons with structured surfaces, Science 305, 5685, pp. 847-848.

[40] Peng, P., Xiao, B. and Wu, Y. (2014). Flat acoustic lens by acoustic grating with curled slits, Physics Letters A 378, 45, pp. 3389-3392.

[41] Sanchez-Hubert, J. and Sanchez-Palencia, E. (1982). Acoustic fluid flow through holes and permeability of perforated walls, Journal of Mathematical Analysis and Applications 87, 2, pp. 427-453.

[42] Simovski, C. R. (2011). On electromagnetic characterization and homogenization of nanostructured metamaterials, Journal of Optics 13, 1, p. 013001.

[43] Suh, K.-D., Ji, C.-H. and Kim, B. H. (2011). Closed-form solutions for wave reflection and transmission by vertical slotted barrier, Coastal Engineering 58, 12, pp. 1089-1096.

[44] Xie, Y., Wang, W., Chen, H., Konneker, A., Popa, B.-I. and Cummer, S. A. (2014). Wavefront modulation and subwavelength diffractive acoustics with an acoustic metasurface, Nature Communications, 5. 
[45] Zhao, J., Ye, H., Huang, K., Chen, Z. N., Li, B. and Qiu, C.-W. (2014). Manipulation of acoustic focusing with an active and configurable planar metasurface transducer, Scientific Reports 4.

[46] Zhao, Y., Engheta, N. and Alù, A. (2011). Homogenization of plasmonic metasurfaces modeled as transmission-line loads, Metamaterials 5, 2, pp. 90-96.

[47] Zhu, J., Chen, Y., Zhu, X., Garcia-Vidal, F. J., Yin, X., Zhang, W. and Zhang, X. (2013). Acoustic rainbow trapping, Scientific Reports 3.

[48] Zhu, J., Christensen, J., Jung, J., Martin-Moreno, L., Yin, X., Fok, L., Zhang, X. and Garcia-Vidal, F. (2011). A holey-structured metamaterial for acoustic deep-subwavelength imaging, Nature Physics 7, 1, pp. 52-55. 